\title{
Wie Wien wächst: Monitoring aktueller Trends hinsichtlich Bevölkerungs- und Siedlungsentwicklung in der Stadtregion Wien
}

\author{
Jakob Eder ${ }^{1} \mathbb{D} \cdot$ Elisabeth Gruber ${ }^{2}$ Peter Görgl ${ }^{2}$ Markus Hemetsberger ${ }^{3}$ \\ Eingegangen: 19. Dezember 2017 / Angenommen: 26. Juni 2018 / Online publiziert: 9. Juli 2018 \\ ๑) Springer-Verlag GmbH Deutschland, ein Teil von Springer Nature 2018
}

\section{Zusammenfassung}

Wie vergleichbare Stadtregionen in Europa ist auch Wien von einem starken Bevölkerungswachstum gekennzeichnet, das teilweise deutlich über den Prognosewerten der 2000er-Jahre liegt. Um die Bevölkerungs- und Siedlungsentwicklung genau beobachten und einordnen zu können, diskutiert der vorliegende Beitrag eine Methodik für ein Monitoring der Stadtregion Wien. Diese stützt sich auf die Analyse weniger, dafür aber zentraler und zeitnah verfügbarer Indikatoren: Bevölkerungsentwicklung, Geburten- und Wanderungsbilanz, Wanderungsströme und Baulandreserven auf kleinräumiger Ebene. Die Ergebnisse des Monitorings zeigen, dass das starke Wachstum der Stadtregion fast ausschließlich auf hohe Wanderungsgewinne aus dem restlichen Österreich und dem Ausland zurückzuführen ist. Diese stiegen in den letzten Jahren deutlich an und konzentrierten sich vor allem auf die Kernstadt. Während folglich eine zunehmende (Re-)Urbanisierung zu beobachten ist, stagniert die Suburbanisierung auf einem hohen Niveau. In der Stadtregion laufen also weiterhin diverse Phasen der Stadtentwicklung parallel ab, wobei manche an Bedeutung gewinnen und manche verlieren. Zusätzlich ist ein sparsamer Umgang mit den Baulandreserven und eine höhere Bebauungsdichte in Zukunft notwendig, um ausreichend Wohnraum für ein weiteres Bevölkerungswachstum in der Stadtregion zur Verfügung stellen zu können. Für zukünftige Monitoring-Projekte sollten erstens zunehmend kleinräumige, registerbasierte Daten für die Analyse von Stadtregionen eingesetzt werden. Zweitens können Szenarien zur zukünftigen Entwicklung der Stadtregion einen wesentlichen Beitrag zur Veranschaulichung der Resultate leisten. Und drittens sollten die Ergebnisse zunehmend in einen internationalen Kontext (sowohl planerisch als auch wissenschaftlich) eingebettet werden.

Schlüsselwörter Stadtregion $\cdot$ Wien $\cdot$ Monitoring $\cdot$ Migration $\cdot$ Siedlungsstruktur $\cdot$ Regionalplanung

Jakob Eder

jakob.eder@oeaw.ac.at

$\triangle$ Dr. Elisabeth Gruber

elisabeth.gruber@univie.ac.at

Peter Görgl

peter.goergl@univie.ac.at

Markus Hemetsberger

markus.hemetsberger@noel.gv.at

1 Institut für Stadt- und Regionalforschung, Österreichische

Akademie der Wissenschaften, Postgasse 7/4/2, 1010 Wien,

Österreich

2 Angewandte Geographie, Raumforschung, Raumordnung, Universität Wien, Universitätsstraße 7, 1010 Wien, Österreich

3 Abteilung Raumordnung und Regionalpolitik,

Amt der Niederösterreichischen Landesregierung,

Landhausplatz 1, 3109 St. Pölten, Österreich 


\title{
How Vienna Grows: Monitoring of Current Trends of Population and Settlement Dynamics in the Vienna Urban Region
}

\begin{abstract}
Like other urban regions in Europe, Vienna has experienced a pronounced population growth that exceeds any previous assumptions. The study at hand discusses a methodology for a monitoring of the urban region, which was generated to assess these developments in detail. It focuses on a few but crucial indicators, namely: population growth, birth and migration rates, migration flows and building land availability on a small scale. The results show that the strong population growth is predominantly caused by high net migration gains from other parts of Austria and foreign countries. These gains have been increasing recently and focused mainly on the core city. Hence, there is a growing tendency towards (re-)urbanisation. In contrast, the numbers of suburbanisation migration are stagnating, although at a high level. Therefore, different phases of urban development can be observed at the same time, though their impact is changing. In addition, a responsible handling of the remaining land reserves and a higher building density are necessary in the future to provide housing for further population growth in the urban region. Future monitoring projects could build upon the following recommendations: First, small-scale and register-based data should be increasingly used for the analysis of urban regions. Second, scenarios for the future development are an effective method to illustrate the results. Third, such findings should be embedded increasingly in an international context, both from a planning and a scientific perspective.
\end{abstract}

Keywords Urban region $\cdot$ Vienna $\cdot$ Monitoring $\cdot$ Migration $\cdot$ Settlement patterns $\cdot$ Spatial planning

\section{Einleitung}

Seit etwa zehn Jahren ist die Stadt Wien von einem Bevölkerungswachstum gekennzeichnet, das jüngst an Dynamik noch zugenommen hat. Bis zum Jahr 2029 rechnet die Stadt mit zwei Millionen Einwohnern (Stadt Wien 2014b: 13). Auch für das Umland wird ein deutlicher Bevölkerungsanstieg erwartet, wodurch die Stadtregion insgesamt in den nächsten Jahren die Einwohnerzahl von drei Millionen Einwohnern erreichen soll (Hanika/Fuchs/Klotz 2015: 133f.). Diese Entwicklung liegt zum Teil deutlich über den

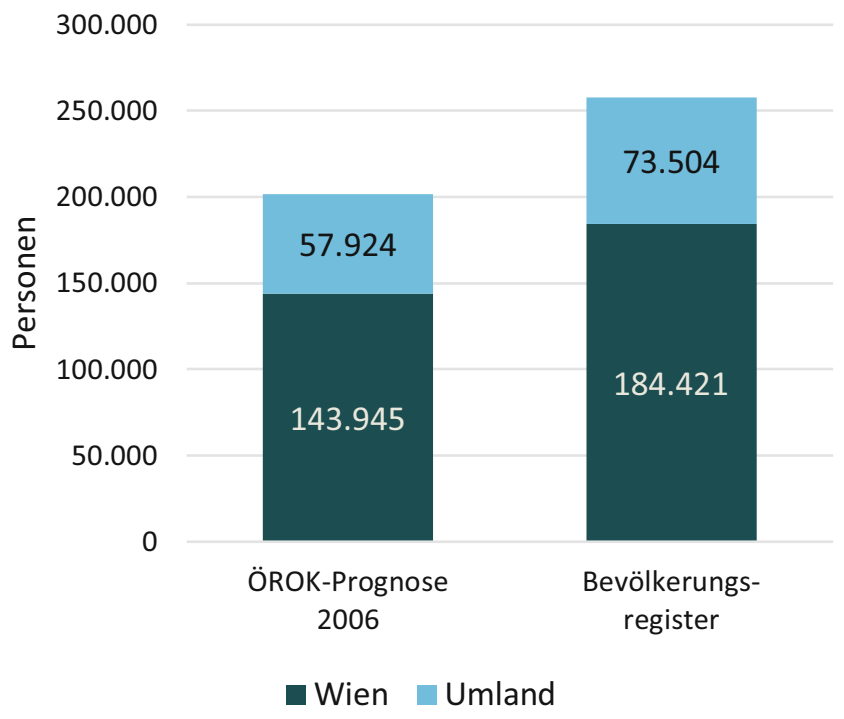

Abbildung 1 Diskrepanz zwischen der ÖROK-Prognose 2006 und dem Bevölkerungsstand 2016 laut Digitalem Bevölkerungsregister der Statistik Austria
Bevölkerungsprognosen der 2000er-Jahre. Beispielsweise war man in der Österreichischen Raumordnungskonferenz (ÖROK) im Jahr 2006 (Hanika 2006: 45f.) von einem Wachstum von 200.000 Personen in der Stadt Wien und den angrenzenden Umlandbezirken bis zum Jahr 2016 ausgegangen. Tatsächlich ist die Bevölkerung in den letzten zehn Jahren jedoch um 260.000 Personen gewachsen, wie aus aktuellen Registerdaten hervorgeht. ${ }^{1}$ Dabei hat vor allem die Stadt Wien eine deutlich dynamischere Entwicklung vollzogen, als dies angenommen worden war (vgl. Abbildung 1).

Diese unerwartete Dynamik in der Bevölkerungsentwicklung hat dazu geführt, dass sich Wissenschaft und Praxis mittlerweile intensiv mit der Beobachtung des Wachstums und dessen Auswirkungen befassen. Während sich eine Vielzahl von wissenschaftlichen Arbeiten (für eine Übersicht vgl. Görgl/Helbich/Matznetter et al. 2011: $110 \mathrm{ff}$.) mit aktuellen Stadtentwicklungsprozessen in der Agglomeration Wien beschäftigt hat, geht es in der raumordnerischen Praxis hauptsächlich um die Frage, inwiefern die Stadt und das Umland dem enormen Wachstum ausreichend Raum zur Verfügung stellen können. Der Zuwachs an Wohnbevölkerung soll auch qualitätsvoll gesteuert werden, um nachteilige Effekte (beispielsweise Zersiedelung im Sinne eines urban sprawl, steigende Verkehrsbelastung oder ausufernden Flächenverbrauch) zu verhindern. Die drei Bundesländer Burgenland, Niederösterreich und Wien haben in diesem Zusammenhang im Rahmen der Planungsgemeinschaft Ost (PGO) bereits mehrere Forschungsprojekte durchgeführt, um über notwendige

\footnotetext{
${ }^{1}$ Vgl. http://www.statistik.at/blickgem/index.jsp (01.06.2018).
} 
Planungsgrundlagen zu verfügen (Fassmann/Görgl/Helbich 2009; Planungsgemeinschaft Ost 2011; Döringer/Görgl/ Huemer 2014). Im Jahr 2015 wurde seitens der Planungsgemeinschaft Ost eine weitere Studie beauftragt, die in der Vergangenheit formulierte Ziele und die beobachteten Entwicklungen einem Monitoring und damit einer Evaluierung unterziehen sollte. Das Monitoring wurde von 2015 bis 2017 vom Institut für Stadt- und Regionalforschung (ISR) der Österreichischen Akademie der Wissenschaften (ÖAW) durchgeführt. Ziel war, eine geeignete Methodik zu entwickeln beziehungsweise Indikatoren zu definieren, die eine regelmäßige Beobachtung der Siedlungsentwicklung ermöglichen. Somit sollte das Monitoring eine aktuelle Grundlage für Ziele und Instrumente der Raumordnung und Raumplanung darstellen. Das rasante Wachstum der Stadt und ihres Umlandes haben dazu geführt, dass eine regelmäBige Analyse in kurzen Zeitabständen sinnvoll erscheint, um räumliche Veränderungsprozesse zeitnah zu erkennen und auf diese reagieren zu können. Zentrales Anliegen des Monitoring-Projekts war zudem die Überprüfung, inwiefern die räumliche Verfügbarkeit von Baulandreserven mit der prognostizierten Bevölkerungsentwicklung korrespondiert.

In diesem Beitrag werden die im Rahmen des PGO-Projekts „Monitoring der Siedlungsentwicklung in der Stadtregion: Strategien zur räumlichen Entwicklung der Ostregion" entwickelte Methodik zum Monitoring der Bevölkerungsentwicklung und Siedlungsstruktur der Stadtregion Wien vorgestellt und diskutiert. Um die Ergebnisse wissenschaftlich zu reflektieren, wurden weiters die aktuell beobachteten Trends in der Stadtregion Wien in den internationalen Forschungsdiskurs eingebettet. In diesem Beitrag werden Teile der detaillierten Ergebnisse aus dem Monitoring vorgestellt (vgl. ausführlich Görgl/Eder/Gruber et al. 2017). Der Projektbericht nimmt jedoch keine Einbettung der Resultate in den aktuellen Diskurs der Raumforschung vor. Dies wird hier geleistet.

Ein weiteres Ziel des vorliegenden Beitrags ist es, einen neuen Schwerpunkt bei der Methodik zur Messung von wachsenden Stadtregionen zu setzen. So werden auf der Basis der Ergebnisse des Projektberichts Szenarien zur Baulandmobilisierung und Bebauungsdichte berechnet, um die Potenziale von geordneter Siedlungsentwicklung und Nachverdichtung darzustellen. Dadurch werden auch die Vorteile von kleinräumigen, registerbasierten Daten verdeutlicht, die bislang nur selten in diesem Kontext zum Einsatz kommen.

Nach einer Beschreibung des aktuellen Forschungsstandes zu Stadtentwicklungsprozessen (Kapitel 2) mit dem Fokus auf Wien und der Berücksichtigung von Beispielen aus anderen Stadtregionen (um später die beobachteten Entwicklungen einordnen zu können), folgt anschließend die detaillierte Beschreibung der Methodik des MonitoringProjektes (Kapitel 3) sowie darauf beruhende Ergebnisse zur Bevölkerungs- und Siedlungsentwicklung in der Stadt- region (Kapitel 4), die mit Szenarien zu Baulandmobilisierung und Bebauungsdichte in Beziehung gesetzt werden (Kapitel 5). In den letzten beiden Kapiteln 6 und 7 finden sich eine Zusammenfassung und Diskussion der Resultate sowie Handlungsempfehlungen.

\section{Historische und aktuelle Trends der Siedlungsentwicklung in der Stadtregion Wien}

Die Beobachtung aktueller stadtregionaler Trends hat in Wien eine lange Tradition (für eine Übersicht vgl. Görgl/ Helbich/Matznetter et al. 2011: 110ff.). Seit den Nachkriegsjahren bis heute hat die Stadt verschiedene Phasen der Transformation erlebt. So sprechen Suitner, Krisch und Pühringer (2018: 17) seit 1850 von einer industriellen Urbanisierung, seit 1910 von einer Schrumpfungsphase, ab dem Jahr 1950 von einer Konsolidierungsphase und seit dem Jahr 2000 von Reurbanisierung. Erst seit Kurzem wachsen Stadt und Umland gemeinsam, zuvor gab es hinsichtlich Bevölkerungswachstum und -rückgang eher gegensätzliche Entwicklungen. Bis in die 1980er-Jahre war die Stadt Wien eine schrumpfende Metropole (Weigl 2003; Oswalt/ Rieniets 2006; Fassmann/Hatz 2009). Der Rückgang der Einwohnerzahl der Kernstadt ließ sich seit der Volkszählung von 1910 (mit einigen Ausnahmen etwa zwischen 1951 und 1961) beobachten. ${ }^{2}$ Während Österreich bis in die 1960erJahre ein Auswanderungsland war, was auch in der Stadtregion Wien spürbar war (Fassmann/Münz 1995), führte seit den 1960er-Jahren vor allem eine negative Binnenwanderungsbilanz dazu, dass die Stadt eine stagnierende beziehungsweise eine negative Bevölkerungsentwicklung verzeichnete.

Eine intensive Siedlungsentwicklung im Rahmen dieser Suburbanisierung konnte vor allem südlich der Stadtgrenze beobachtet werden (vgl. Görgl 2008). In den 1990erJahren breitete sich die verstärkte Bautätigkeit auch in jene Umlandgemeinden aus, die etwas weiter von der Kernstadt entfernt waren. Tendenzen der Suburbanisierung ließen sich auch jenseits der zentralen Verkehrsachsen beobachten, wo das Bauland noch günstiger und vor allem verfügbar war (vgl. Görgl/Helbich/Matznetter et al. 2011). Nicht nur eine Zunahme der Wohnbevölkerung, sondern auch eine Verlagerung der wirtschaftlichen Aktivität konnte beobachtet werden. Die Entwicklung von der Suburbia zur Postsuburbia, also das zeitgleiche Auftreten verschiedener Entwicklungen wie Fragmentierung, Verdichtung an den Rändern, diversifizierte Bevölkerung und eine polyzentrische Struktur (Helbich 2012: 40), lässt sich im Wiener Umland anhand von verschiedenen Entwicklungen beobachten (vgl.

\footnotetext{
${ }^{2}$ Vgl. http://www.statistik.at/blickgem/index.jsp (01.06.2018).
} 
Görgl 2008; Helbich/Leitner 2009; Helbich/Leitner 2010; Helbich 2012; Görgl/Gruber 2015). Dennoch verzeichnen die meisten Umlandgemeinden nach wie vor ein stetiges Anwachsen der Bevölkerungszahl.

In den letzten Jahren ist allerdings auch ein dynamisches Bevölkerungswachstum in der Stadt Wien zu beobachten: sowohl in den Stadterweiterungsgebieten als auch in der dicht bebauten Kernstadt. Dieser Trend wird im deutschsprachigen Raum unter dem Begriff Reurbanisierung subsummiert und ist dort seit gut zehn Jahren auch in anderen Metropolen zu beobachten (vgl. Brake/Herfert 2012). Bereits in den 1980er-Jahren wurde das Konzept der Reurbanisierung als potenzielle Phase der Stadtentwicklung von van den Berg, Drewett, Klaassen et al. (1982: 36) beschrieben. Diese folgt auf die Suburbanisierung und wird als Trendumkehr verstanden, da die Kernstadt wieder ein Bevölkerungswachstum erlebt. Nachdem dieses Phänomen in den letzten Jahrzehnten tatsächlich in fast allen Großstädten beobachtet werden konnte, haben verschiedene Autoren versucht, eine Erklärung für diese Reurbanisierung zu finden, wie beispielsweise den Einfluss von demographischen Prozessen (Cheshire 1995; Lever 1993) oder die Bedeutung von Migrationsbewegungen (Hirschle/Schürt 2010; Herfert/ Osterhage 2012). Allgemein können veränderte Präferenzen von Haushalten und neue Familienformen sowie die zunehmende Bedeutung internationaler Migration als Begründung für das Wachstum von Städten herangezogen werden (Buzar/Hall/Ogden 2007: 65 f.). Reurbanisierung lässt sich auf eine Veränderung von Lebensstilen zurückführen, etwa die zunehmende Anzahl an kinderlosen, karriereorientierten Paaren. Auch führt ein Hinauszögern von Geburten und Heirat dazu, dass Wohnformen wie das Einfamilienhaus erst zu einem späteren Zeitpunkt nachgefragt und in der Zwischenzeit kleinere und zentralere Wohnformen bevorzugt werden. Bedarf nach kleineren Wohneinheiten gibt es aber auch bei der Altersgruppe der älteren und hochbetagten Personen. Und schließlich gilt auch die Zunahme von internationaler Migration als Katalysator für das urbane Wohnen (Rérat 2012).

Die Reurbanisierung trat international - etwa in Frankreich und den USA - bereits ab den 1990er-Jahren auf (Ogden/Hall 2000; Champion 2001; Rérat 2012). Die gesteigerte Attraktivität des urbanen Wohnens wurde in diversen Studien vor allem mit einer allgemeinen Qualitätssteigerung und konkreten Projekten der Stadterneuerung und -entwicklung (etwa von früheren Industriearealen) in Verbindung gebracht (Rérat 2012). Stadtaufwertung und Gentrifizierung können somit als Prozesse identifiziert werden, die zu einer zunehmenden Nachfrage nach Wohnungen in der Innenstadt geführt haben.

Neben der Reurbanisierung ist Suburbanisierung im Wiener Umland weiterhin auf hohem Niveau zu beobachten, da sich die Stadtumlandbereiche ebenfalls dynamisch entwickeln. Durch die Gleichzeitigkeit von Konzentration und Dezentralisierung und das Nebeneinander komplexer funktionaler und sozioökonomischer Strukturen kann die Stadtregion Wien insgesamt auch als eine Variante der „Postmetropolis“ bezeichnet werden (Soja 2000; Helbrecht 2001). Das Herausbilden einer dynamischen Stadtregion mit einer ausgeprägten Suburbanisierung im Wiener Umland hat bereits in der Vergangenheit dazu geführt, dass raumordnungspolitische Fragestellungen für die Stadtregion immer stärker aus einer holistischen Perspektive betrachtet werden mussten. In diesem Zusammenhang wurde die Planungsgemeinschaft Ost (PGO) bereits in den 1970er-Jahren als gemeinsame Organisation der Länder Wien, Niederösterreich und Burgenland damit betraut, raumplanerisch relevante Fragen in der österreichischen Ostregion zu analysieren, abzustimmen und zu koordinieren. Eine gemeinsame Planungsperspektive zwischen den Gemeinden ist aufgrund der Konkurrenz um Einwohner und Unternehmen, welche sich auf die Mittelzuweisung direkt auswirken, schwierig. Neben dem Konkurrenzverhalten sind es unterschiedliche Interessenlagen, (fachliche) Kapazitäten und nicht zuletzt auch politische Animositäten, die dafür sorgen können, dass - im Prinzip - einfache interkommunale Absprachen und der Blick über die Gemeindegrenzen erschwert werden (Görgl/Gruber 2015).

Im Fall von Wien und seinem Umland gibt es die Besonderheit, dass hier nicht nur Gemeinde-, sondern auch Bundesländergrenzen verlaufen. Dies und eine ,traditionelle politische Polarität' stellen eine zusätzliche Herausforderung für Kooperationen zwischen den Umlandgemeinden und der Bundeshauptstadt dar. Das Fehlen einer Bundeskompetenz für Raumordnung in Österreich macht es aber notwendig, dass eine gemeinsame Absprache erfolgt. Weiters ist die zunehmende Bedeutung von Städten und urbanen Konzentrationen in den Kontext der globalen Wirtschaftsentwicklung einzubetten (vgl. Sassen 1994), was sich seit den 1990er-Jahren verstärkt auch in Wien beobachten lässt - einerseits durch den Beitritt Österreichs zur EU, andererseits durch den Fall des Eisernen Vorhanges und die EUOsterweiterung (Stadt Wien 2014a: 9). Ein weiterer Beleg dafür ist die Europaregion Centrope ${ }^{3}$, mit Hilfe derer seit 2003 die transnationale Bedeutung dieses Raums auf internationaler Ebene betont werden soll (Giffinger/Hamedinger 2009: 7).

Durch den zunehmenden Leidensdruck der Gemeinden, der fortschreitenden Knappheit von Ressourcen und der steigenden Anzahl an Einwohnern und den sich daraus ergebenden Herausforderungen wächst die Notwendigkeit einer gemeinsamen Betrachtung respektive Planung der Entwicklung von Stadtregionen. Im Rahmen des Projekts „Strate-

\footnotetext{
${ }^{3}$ https://www.wien.gv.at/wirtschaft/eu-strategie/centrope.html (01.06.2018).
} 
gien zur räumlichen Entwicklung der Ostregion (SRO)“ im Jahr 2008 wurden der Stadtregion gute Voraussetzungen für die erfolgreiche Bewältigung des Wachstums attestiert, vor allem, weil ausreichend Flächenreserven vorhanden waren. Dennoch wurde darauf hingewiesen, dass geeignete siedlungspolitische Maßnahmen das Wachstum dorthin leiten müssten, wo es strukturell und ökologisch sinnvoll ist (Fassmann/Görg1/Helbich 2009: 181). Als Grundprinzip für die räumliche Entwicklung wurde das Leitbild der strukturierten Stadtregion entworfen, dessen Leitmotiv ein möglichst geordnetes und nachhaltiges Wachstum ist. Als Ziele zur Standortentwicklung wurden unter anderem die Konzentration und der Ausbau von regionalen Entwicklungszentren entlang eines (auszubauenden) hochrangigen Verkehrsnetzes, der Erhalt dörflicher Strukturen in den Achsenzwischenräumen, die Einschränkung von Siedlungsentwicklung in landschaftlich sensiblen Gebieten und der Erhalt abgegrenzter und kompakter Siedlungsstrukturen definiert (Planungsgemeinschaft Ost 2011: 8). Der Prozess des Monitorings sollte nun acht Jahre später überprüfen, inwiefern diese planerischen Grundprinzipien, die weitgehend einem punktaxialen Entwicklungsleitbild entsprechen, eingehalten werden konnten. Daraus leitet sich weiters die Frage $a b$, inwiefern für die Zukunft neue Prinzipien der stadtregionalen Entwicklung formuliert werden müssen, um die Hindernisse der Kooperation leichter bewältigen zu können.

\section{Methodik}

In der deutschsprachigen (Weiland/Richter 2008; Othengrafen/Schmidt-Lauber/Hannemann et al. 2016; Pohlan/Strote 2017), aber auch in der internationalen Literatur (Banerjee 1996; Yueliang/Ruisong 2010; Kontgis/Schneider/Fox et al. 2014) hat sich in den letzten Jahren der Begriff „Monitoring " für eine systematische, regelmäßige Analyse von Siedlungsregionen etabliert. Während in Schwellenländern hauptsächlich Satellitenbilddaten eingesetzt werden, um die Siedlungsentwicklung einer Stadtregion zu analysieren (Banerjee 1996; Yueliang/Ruisong 2010; Kontgis/ Schneider/Fox et al. 2014), kommen im europäischen Raum zunehmend quantitative Daten aus der amtlichen Statistik zum Einsatz (Weiland/Richter 2008; Pohlan/Strote 2017). Dafür ausschlaggebend ist einerseits die Datenverfügbarkeit, die in westlichen Ländern immer besser wird. Andererseits verfolgen solche Monitoring-Projekte häufig konkrete Fragestellungen, die sich mittels Fernerkundung allein nicht beantworten lassen. Die verwendeten Indikatoren sind dabei oft hochspezialisiert und ermöglichen präzise Aussagen. Aufgrund des hohen Aufwands bei der Datenerhebung und -aufbereitung ist eine erneute Durchführung des Monitorings in vielen Fällen aber dennoch mit einem erheblichen Ressourceneinsatz verbunden.
Die Abwägung zwischen einem adäquaten Ressourceneinsatz und dem Ziel aussagekräftiger Ergebnisse hat auch im vorliegenden Monitoring eine tragende Rolle gespielt. Die Methodik des Monitorings im Forschungsprojekt wird im folgenden Abschnitt vorgestellt. Danach wird die Vorgehensweise zur Berechnung der Szenarien zu Baulandmobilisierung und Bebauungsdichte im Rahmen des vorliegenden Beitrags diskutiert, die auf den Ergebnissen des Monitorings aufbaut.

\subsection{Monitoring der Stadtregion Wien}

Für das diesem Beitrag zu Grunde liegende MonitoringProjekt wurde eine Abgrenzung der Stadtregion gewählt, die bereits bei vorangegangenen Projekten der Planungsgemeinschaft Ost zum Einsatz kam (vgl. Abbildung 2). Diese folgt sowohl einem pragmatischen als auch einem politischplanerischen Zugang, um auch die Verantwortlichkeit der aneinandergrenzenden Bundesländer $\mathrm{zu}$ berücksichtigen (Görgl/Helbich/Matznetter et al. 2011: 109f.; Planungsgemeinschaft Ost 2011: 8) und ist nicht deckungsgleich mit Studien, die den Verflechtungsraum anders auslegen (z. B. Giffinger/Kalasek/Wonka 2006). Die PGO-Abgrenzung verfolgt das Ziel, die Ausdehnung der Stadtregion über funktionale Verflechtungen und die Berücksichtigung der existierenden ,stadtregionalen Wachstumspole' (beispielsweise Bezirkshauptstädte) zu definieren. Die Stadtregion sollte demzufolge aus Einheiten bestehen, die miteinander wirtschaftlich und/oder aktionsräumlich verbunden sind. Neben der Kernstadt Wien wurden so 205 Gemeinden aus Niederösterreich und 63 aus dem Burgenland im Untersuchungsgebiet der Stadtregion Wien zusammengefasst, die im Rahmen dieser Studie einem Monitoring unterzogen wurden.

Der Monitoring-Prozess der Siedlungsentwicklung in der Stadtregion Wien inkludiert unterschiedliche Methoden, die verschiedene Perspektiven auf die Thematik ermöglichen. Die Vorgehensweise wurde in zahlreichen Workshops mit den Auftraggebern und externen Experten festgelegt und ist in Abbildung 3 schematisch dargestellt. Vorrangiges Ziel ist es, ein übersichtliches Datenset auf der Basis des Bevölkerungsregisters zu definieren, das in regelmäBigen, kurzen Abständen verfügbar und für nachfolgende Untersuchungen unkompliziert zugänglich ist. Auf einige Spezialindikatoren muss so zwar verzichtet werden, dafür bleibt das Monitoring flexibel genug, um auf kurzfristige Trends reagieren zu können. Um die Dynamiken in der Stadtregion umfassend zu beschreiben, wird auf aktuelle quantitative Daten zurückgegriffen, welche von den statistischen Ämtern auf kleinräumiger Ebene zur Verfügung gestellt wurden. Dazu zählen die Bevölkerungsentwicklung, die natürliche Bevölkerungsbewegung, Wanderungsströme und die Baulandreserven. Diese Parameter sollten in jedem 
Abbildung 2 Abgrenzung der Stadtregion Wien auf der Basis der Planungsgemeinschaft Ost (PGO)

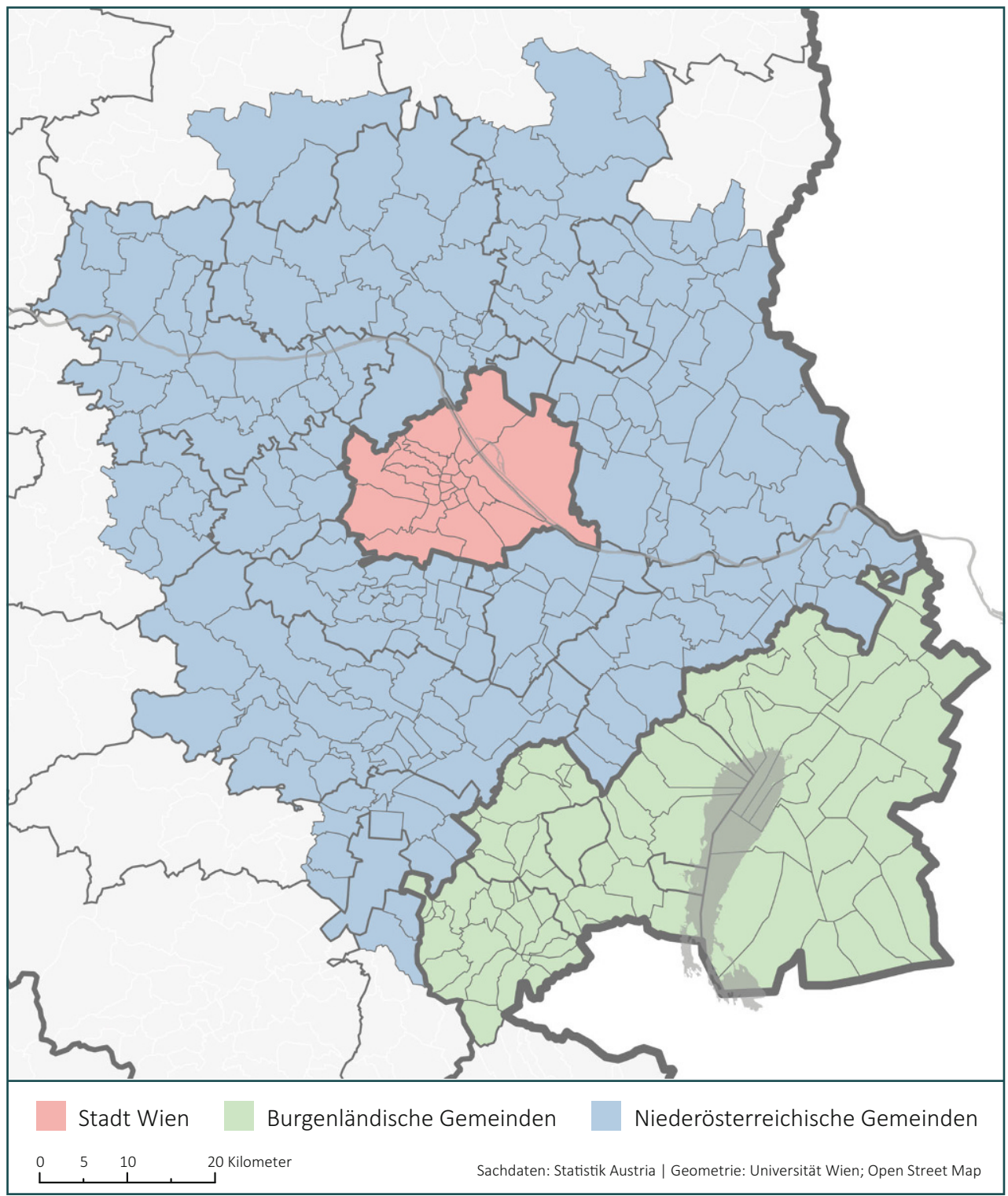

zukünftigen Monitoring berücksichtigt werden. Kommt es zu außergewöhnlichen Entwicklungen (beispielsweise wie der Weltwirtschaftskrise 2008 oder den Flüchtlingsbewegungen 2015), können Sonderthemen in das Indikatorenset (beispielsweise die detaillierte Analyse bestimmter Wanderungssalden) aufgenommen werden. Im vorliegenden Beitrag wird auf die Bevölkerungsdynamik (Themenblock 1 vgl. Kapitel 4), die Siedlungsentwicklung (Themenblock 2 - vgl. Kapitel 5.1) und die Baulandreserven (Themenblock 3 - vgl. Kapitel 5.2) eingegangen.

Neben der Auswertung von Daten der Gemeindeebene wird in einem weiteren Schritt auf das regionalstatistische
Raster ${ }^{4}$ zurückgegriffen, um die Bevölkerungsentwicklung der letzten Jahre noch präziser unabhängig von administrativen Grenzen beschreiben zu können. Eingesetzt wird ein Raster mit einer Kantenlänge von 1.000 Metern, wobei im Rahmen dieser Untersuchung für jede Rasterzelle die absolute Bevölkerungszahl von 2010 und 2015 zur Verfügung stand. Dieses Raster wird in zwei Schritten mit weiteren Informationen angereichert. Erstens erfolgt die Verschneidung in einem Geographischen Informationssystem (GIS) mit den Baulandreserven auf der Gemeindeebene. In einem weiteren Schritt werden erreichbarkeitsbasierte Potenzialberechnungen durchgeführt, die die Darstellung der Unter-

\footnotetext{
${ }^{4}$ Regionalstatistische Rastereinheiten der Statistik Austria; vgl. http:// www.statistik.at/web_de/klassifikationen/regionale_gliederungen/regio nalstatistische_rastereinheiten/index.html (01.06.2018).
} 


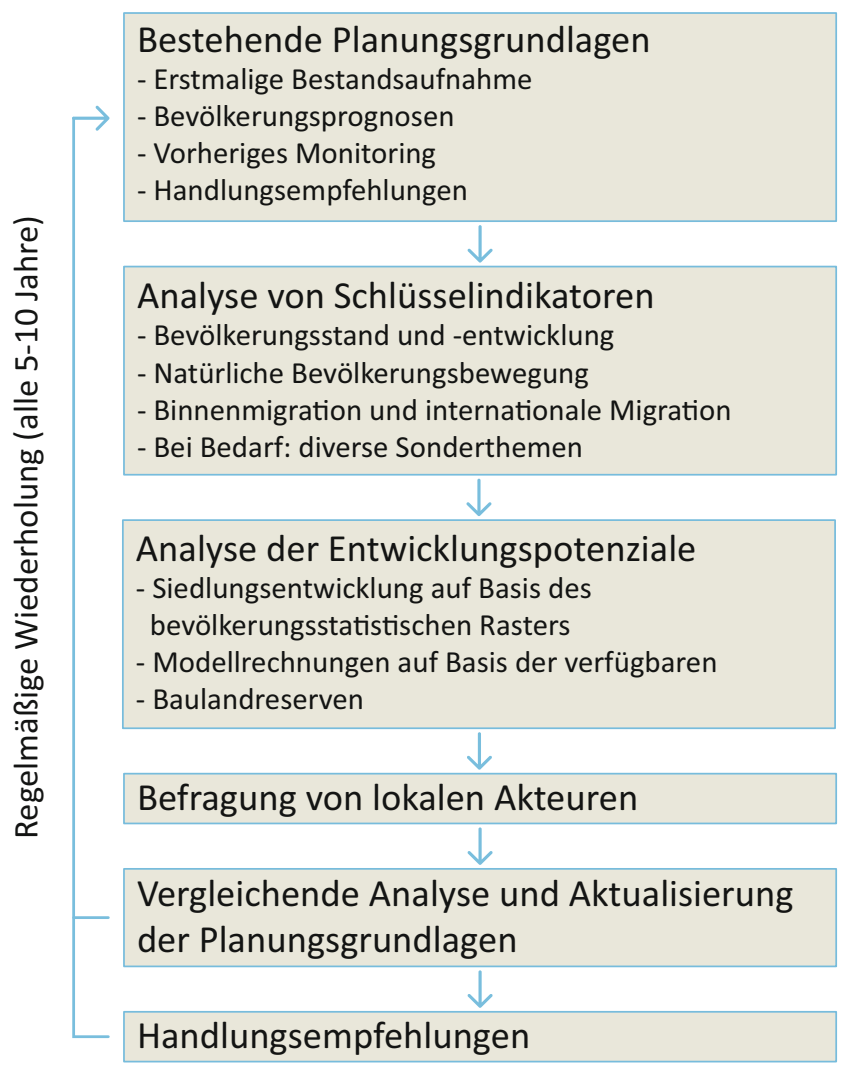

Abbildung 3 Schematischer Ablauf und Schlüsselindikatoren des Monitorings

schiede in der Ausstattungsqualität verschiedener Raumeinheiten ermöglichen.

Die Potenziale werden unabhängig von Verwaltungsgrenzen für 1.000-Meter-Rasterzellen berechnet und können somit direkt miteinander verglichen werden. Ihre Berechnung beruht auf zwei Säulen: einerseits auf den Standorten von Einrichtungen der Daseinsvorsorge (z. B. Bildung, Nahversorgung, Gesundheit, Betreuung), andererseits auf der Erreichbarkeit dieser Standorte von einer Rasterzelle aus. Die Standorte werden dabei auf die Rasterzellen umgelegt. Die Fahrzeiten werden im motorisierten Individualverkehr auf der Basis von „OpenStreetMap“5, im öffentlichen Verkehr anhand von Fahrplandaten des Verkehrsverbundes Ostregion (VOR) berechnet und liegen demnach als Fahrzeitmatrizen vor.

Mit den einzelnen Standorten werden sogenannte Erreichbarkeitsindikatoren gebildet, beispielsweise die Anzahl der innerhalb von 30 Minuten Fahrzeit im motorisierten Individualverkehr erreichbaren Schulen. Die reine Anzahl von erreichbaren Standorten wird noch mittels verschiedener Funktionen modelliert, da etwa die Erreichbarkeit eines sechsten Supermarkts das Potenzial für den Endverbraucher nicht mehr zusätzlich erhöht oder die Bedeu-

\footnotetext{
5 Vgl. https://www.openstreetmap.org (01.06.2018).
}

tung eines Standortes für die Ausstattungsqualität mit zunehmender Entfernung abnimmt. Um die Entwicklungspotenziale der Stadtregion zu ermitteln, werden 19 Einzelindikatoren modelliert, zueinander gewichtet, normiert und zusammengeführt. Das Ergebnis ist ein Potenzialwert für jede Rasterzelle von 0 für „kein Potenzial“ bis 100 für das höchste Potenzial in der gesamten betrachteten Region. Somit lässt sich mit einer sehr hohen räumlichen Auflösung beurteilen, wo und in welchen Potenzialkategorien die Stadtregion Wien gewachsen ist, und im Weiteren, ob ein Wachstum in der Fläche oder eine Konzentration zu beobachten ist.

Aufgrund der quantitativen Ergebnisse wurden im Rahmen dieses Monitorings auch noch Beispielgemeinden ausgewählt, in denen mit Gemeindevertretern die aktuellen Entwicklungen und Planungsherausforderungen besprochen wurden. Auf die qualitativen Ergebnisse des Monitorings wird an dieser Stelle nicht eingegangen, da der Schwerpunkt des Beitrags auf der Bewertung der quantitativen Bevölkerungs- und Siedlungsentwicklung sowie der Szenarienentwicklung liegt. ${ }^{6}$

\subsection{Szenarien zur Baulandmobilisierung und Bebauungsdichte}

Zusätzlich werden im Rahmen des vorliegenden Beitrags die Baulandreserven in einer Modellrechnung der Bevölkerungsentwicklung gegenübergestellt, wodurch der potenzielle zukünftige Siedlungsflächenverbrauch als wichtige Größe der Siedlungsentwicklung betont wird. Dazu werden, wie in vorherigen Arbeiten zur Stadtregion Wien, die in einer Studie des Salzburger Instituts für Raumordnung und Wohnen (2007: 9) für unterschiedliche Bebauungsformen ermittelten Wohneinheiten je Hektar mit der durchschnittlichen Haushaltsgröße in der Stadtregion Wien multipliziert, die bei einem Wert von knapp über zwei liegt (Görgl/Eder/ Gruber et al. 2017: $45 \mathrm{ff}$.). So erhält man eine hypothetische Zahl an Einwohnern je Hektar, die - multipliziert mit den Baulandreserven - Aufschluss darüber gibt, für wie viele Personen Wohnraum bei einer unterschiedlich dichten Bebauung zur Verfügung stehen würde. Zusätzlich werden unterschiedliche Potenziallagen und Baulandmobilisierungsszenarien berücksichtigt. Durch diese Annäherung wird es möglich, Aussagen darüber zu treffen, ob die derzeitig verfügbaren Baulandreserven für das zukünftig zu erwartende Bevölkerungswachstum ausreichend sind, oder ob weitere Ausweisungen sinnvoll sind.

\footnotetext{
${ }^{6}$ Vgl. die Ergebnisse des qualitativen Teils des Monitorings, beispielsweise zur Einschätzung der Treffsicherheit der verfügbaren Planungsinstrumente, bei Görgl/Eder/Gruber et al. (2017).
} 


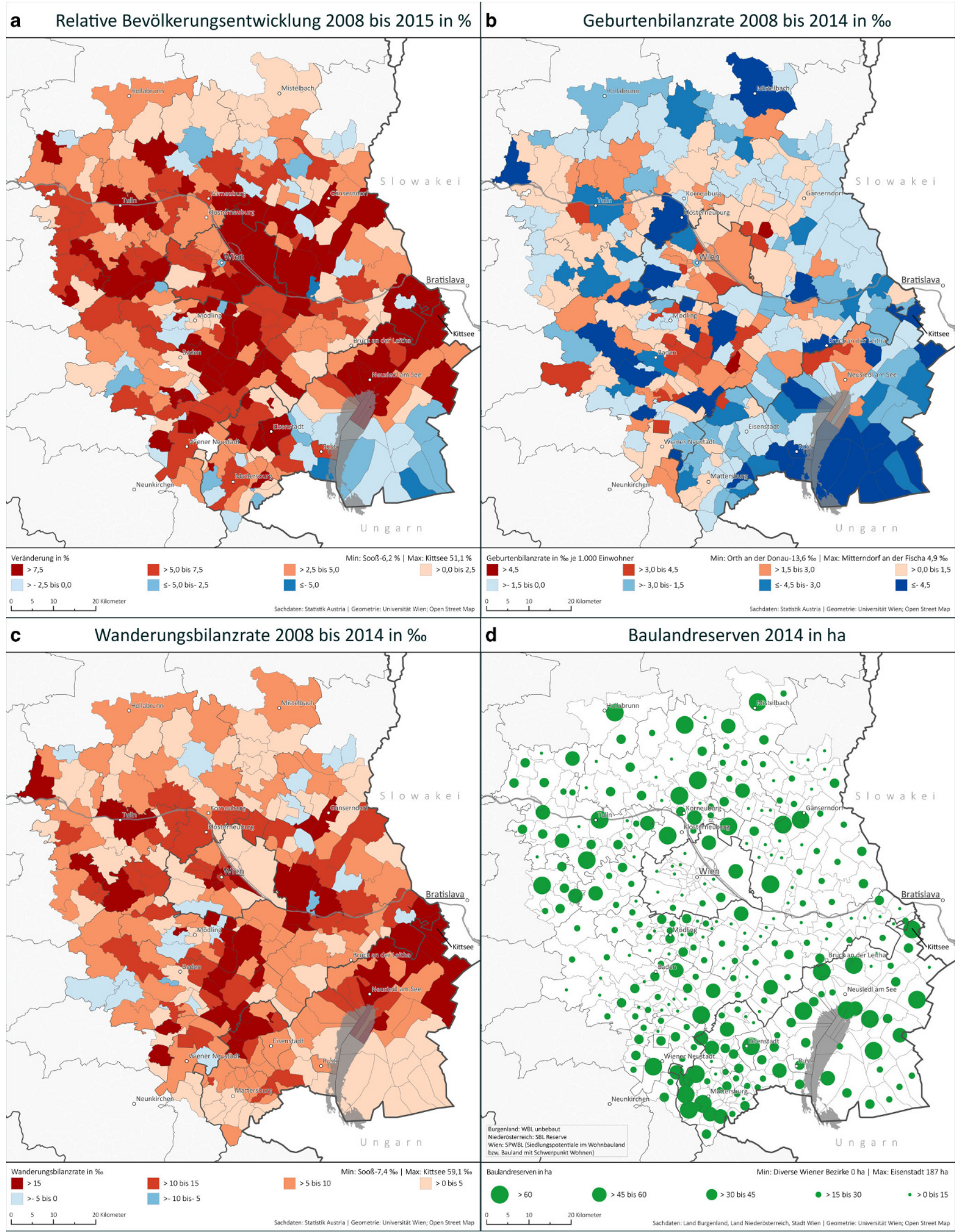

Abbildung 4 Überblick über die zentralen Ergebnisse des Monitorings. Quelle: Görgl/Eder/Gruber et al. $(2017: 39,44,64,86)$ 
Tabelle 1 Bevölkerungsentwicklung in der Stadtregion Wien 2002 bis 2015 (Der Beginn des Vergleichszeitraumes wurde mit 2002 festgelegt, da das Bevölkerungsregister erst seit 2002 zur Verfügung steht und die Registerdaten mit jenen der Volkszählung 2001 nicht direkt vergleichbar sind)

\begin{tabular}{|c|c|c|c|c|c|c|c|}
\hline & & & & Durchsc & e jährlic & vicklung & \\
\hline & Bevölkerun & & & $2002 \mathrm{bi}$ & & $2008 \mathrm{bis}$ & \\
\hline & 2002 & 2008 & 2015 & absolut & relativ & absolut & relativ \\
\hline SRO & 2.435 .674 & 2.587 .562 & 2.763 .761 & 25.315 & $1,0 \%$ & 25.171 & $0,9 \%$ \\
\hline Wien & 1.571 .123 & 1.671 .221 & 1.797 .337 & 16.683 & $1,0 \%$ & 18.017 & $1,0 \%$ \\
\hline Umland & 864.551 & 916.341 & 966.424 & 8.632 & $1,0 \%$ & 7.155 & $0,8 \%$ \\
\hline Nördlicher Teil & 329.947 & 352.609 & 372.832 & 3.777 & $1,1 \%$ & 2.889 & $0,8 \%$ \\
\hline Südlicher Teil & 534.604 & 563.732 & 593.592 & 4.855 & $0,9 \%$ & 4.266 & $0,7 \%$ \\
\hline
\end{tabular}

Quelle: Digitales Bevölkerungsregister der Statistik Austria (STATcube), Tabelle „Bevölkerung zu Quartalsbeginn ab 2002“; eigene Berechnungen

\section{Aktuelle demographische Entwicklungen in der Stadtregion Wien}

In diesem Kapitel sind zentrale Ergebnisse des Monitorings zusammengefasst, um einen Überblick über die aktuellen Entwicklungen in der Stadtregion Wien zu geben. Diese orientieren sich an den wesentlichen Indikatoren, die in der Methodik festgelegt wurden. Die wichtigsten Trends sind kartographisch in Abbildung 4 zusammengefasst und werden in den jeweiligen Abschnitten detailliert beschrieben.

\subsection{Bevölkerungsentwicklung}

Die Stadtregion Wien ist im Untersuchungszeitraum 2008 bis 2015 um knapp 180.000 Personen gewachsen, was im jährlichen Durchschnitt etwa 25.000 Personen oder 0,9 \% entspricht. Damit liegt man in etwa auf dem Niveau des Vergleichszeitraumes 2002 bis 2008 (vgl. Tabelle 1). Deutlich wird, dass die Stadtregion zwar insgesamt wächst, der überwiegende Teil des Wachstums jedoch in der Stadt Wien stattfindet. So entfallen zwischen 2008 und 2015 über zwei Drittel des Wachstums auf Wien $(71,6 \%)$ und nur $28,4 \%$ auf die umliegenden Gemeinden. Im Zeitraum 2002 bis 2008 lag dieses Verhältnis noch bei 65,9\% für Wien und $34,1 \%$ für das Umland.

Anhand dieser Ergebnisse lässt sich erkennen, dass Reurbanisierung der bestimmende Trend in der Stadtregion Wien ist. Nur die Kernstadt Wien kann das durchschnittliche jährliche Wachstum von 1,0 \% halten, in den anderen Teilräumen geht es leicht zurück. Das ist insofern bemerkenswert, weil die Kernstadt Wien bis Anfang der 2000erJahre in vielen Bezirken einen Bevölkerungsrückgang verzeichnete (Fassmann/Görg1/Helbich 2009: 55), nun aber alle Bezirke (mit Ausnahme der historischen Innenstadt, dem 1. Bezirk) ein starkes Bevölkerungswachstum aufweisen.

Abbildung 4a zeigt das räumliche Muster der Bevölkerungsentwicklung 2008 bis 2015. Im Großen und Ganzen verzeichnen alle Gemeinden und Wiener Gemeindebezirke ein zum Teil starkes Bevölkerungswachstum, nur ein- zelne Gemeinden weisen leichte Rückgänge auf. Ausnahme bildet dabei der burgenländische Seewinkel im Südosten der Stadtregion, der durchgängig von einem Rückgang geprägt ist. Neben den Bevölkerungsgewinnen entlang der zentralen Verkehrsachsen Richtung Westen, Süden und Norden lässt sich noch ein anderes interessantes Phänomen in der Stadtregion beobachten. Das starke Wachstum in einigen Gemeinden (beispielsweise Kittsee: 51,1\%) im Osten der Stadtregion lässt sich auf eine grenzüberschreitende Suburbanisierung von Bratislava ausgehend zurückführen. Aktuelle Siedlungsentwicklungen im Agglomerationsraum der slowakischen Hauptstadt haben also auch Auswirkungen auf die Stadtregion Wien (Hardi 2012; Sohn/Giffinger 2015).

Aus Abbildung 5 sind die Auswirkungen der Weltwirtschaftskrise nach 2008 auf die Bevölkerungsentwicklung ersichtlich, die vergleichsweise niedrige Wanderungsgewinne und damit ein geringeres Wachstum zur Folge hatte. Bereits ab 2007 sinken die Wachstumsraten, bevor sie ab 2012 wieder deutlich ansteigen. Seit diesem Zeitpunkt wächst auch die Kernstadt überdurchschnittlich schnell und deutlich dynamischer als die anderen Teilräume der Stadtregion. Relativ betrachtet ist der nördliche Teilraum stärker gewachsen als der südliche, davon war man aufgrund der niedrigeren Bodenpreise im Norden Wiens auch ausgegangen (Fassmann/Görgl/Helbich 2009: 117). Die absoluten Zahlen (vgl. Tabelle 1) unterstreichen jedoch, dass nach wie vor die Südachse für viele Personen attraktiver ist.

\subsection{Bilanz der Geburten und Sterbefälle sowie der Wanderungen}

Die detaillierte Betrachtung der wesentlichen Komponenten der Bevölkerungsentwicklung zeigt ein für europäische Stadtregionen typisches Bild. Die Bilanz aus Geburten und Sterbefälle trägt in der Stadtregion Wien mittlerweile kaum noch zum Wachstum bei $(4,0 \%)$, Bevölkerungsgewinne gehen fast ausschließlich auf die positive Wanderungsbilanz zurück $(96,0 \%)$ (vgl. Tabelle 2). Dies bestätigt sich auch in Abbildung $4 \mathrm{~b}$ und $4 \mathrm{c}$. Eine positive Bilanz der natürlichen 
Abbildung 5 Bevölkerungsentwicklung in der Stadtregion Wien 2002 bis 2015. Quelle: Digitales Bevölkerungsregister der Statistik Austria (STATcube), Tabelle „Bevölkerung zu Quartalsbeginn ab 2002“; eigene Berechnungen

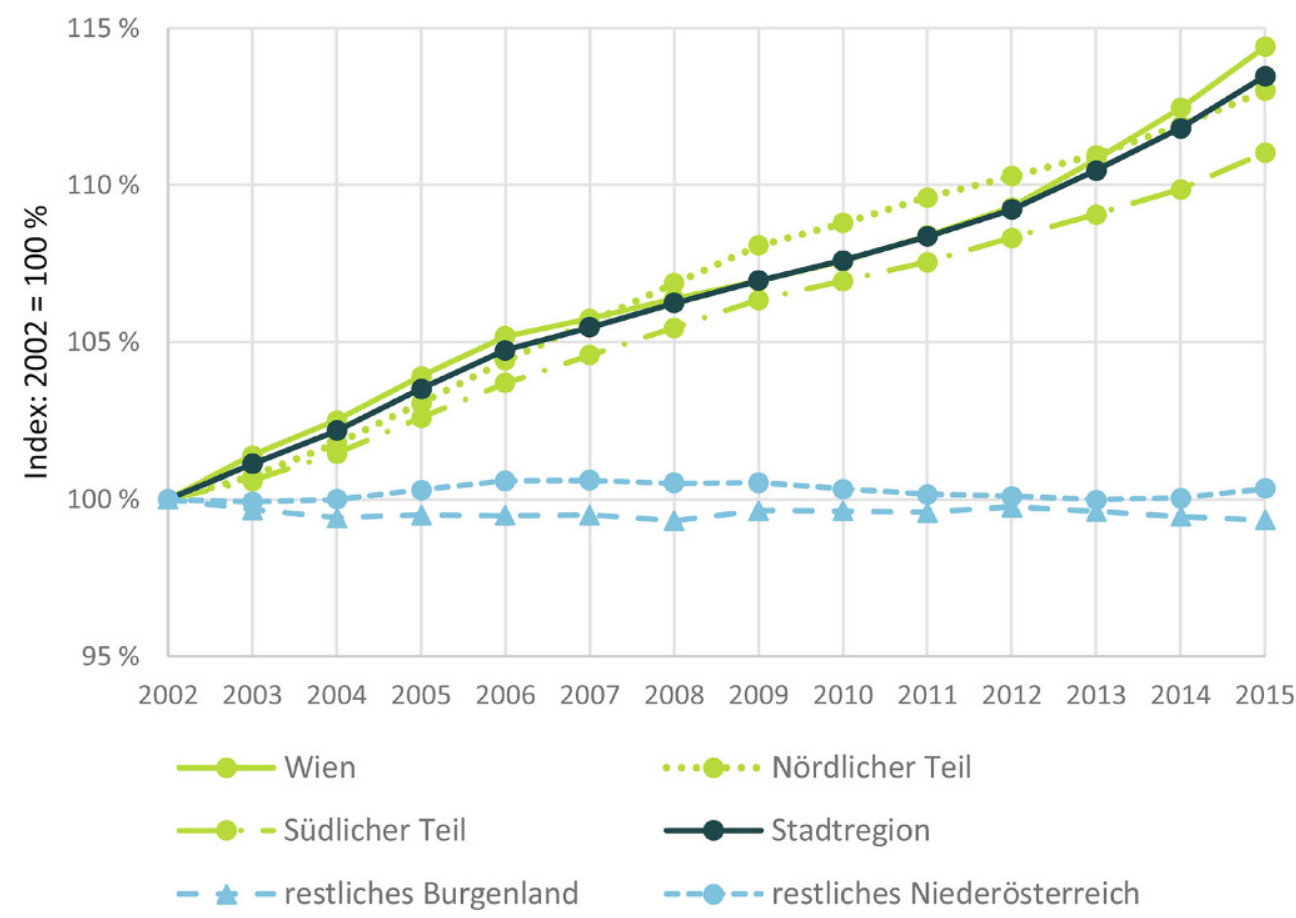

Tabelle 2 Bilanz der Geburten und Sterbefälle sowie der Wanderungen in der Stadtregion Wien 2008 bis 2014 (Die Bilanzen konnten nur bis 2014 ausgewertet werden, da die Wanderungsstatistik 2015 zum Zeitpunkt des Monitorings noch nicht zur Verfügung stand)

\begin{tabular}{|c|c|c|c|c|c|c|c|c|}
\hline & \multicolumn{8}{|c|}{ Bilanz aus Geburten- und Sterbefällen } \\
\hline & 2008 & 2009 & 2010 & 2011 & 2012 & 2013 & 2014 & Gesamt \\
\hline SRO & 715 & -389 & 895 & 1.360 & 400 & 912 & 2.322 & 6.935 \\
\hline Wien & 1.673 & 822 & 1.702 & 2.252 & 1.861 & 2.359 & 3.246 & 16.291 \\
\hline Umland & -958 & -1.211 & -807 & -892 & -1.461 & -1.447 & -924 & -9.356 \\
\hline Nördlicher Teil & -402 & -430 & -235 & -408 & -546 & -522 & -300 & -3.589 \\
\hline \multirow[t]{3}{*}{ Südlicher Teil } & -556 & -781 & -572 & -484 & -915 & -925 & -624 & -5.767 \\
\hline & \multicolumn{8}{|c|}{ Wanderungsbilanz } \\
\hline & 2008 & 2009 & 2010 & 2011 & 2012 & 2013 & 2014 & Gesamt \\
\hline SRO & 17.089 & 15.538 & 17.527 & 18.969 & 29.835 & 31.538 & 37.255 & 167.751 \\
\hline Wien & 7.445 & 8.878 & 10.977 & 11.821 & 22.314 & 22.711 & 26.692 & 110.838 \\
\hline Umland & 9.644 & 6.660 & 6.550 & 7.148 & 7.521 & 8.827 & 10.563 & 56.913 \\
\hline Nördlicher Teil & 4.349 & 2.754 & 2.915 & 2.615 & 2.703 & 3.637 & 3.834 & 22.807 \\
\hline Südlicher Teil & 5.295 & 3.906 & 3.635 & 4.533 & 4.818 & 5.190 & 6.729 & 34.106 \\
\hline
\end{tabular}

Quelle: Digitales Bevölkerungsregister der Statistik Austria (STATcube), Tabellen „Gestorbene“ und „Geborene“ sowie „Wanderungen innerhalb Österreichs“ und „Wanderungen mit dem Ausland“; eigene Berechnungen

Bevölkerungsentwicklung kommt hauptsächlich in einigen Wiener Gemeindebezirken und entlang der Südachse vor, wohingegen fast die gesamte Stadtregion Wanderungsgewinne verzeichnet.

Im Zuge der Wirtschaftskrise war die Bilanz der natürlichen Bevölkerungsentwicklung 2009 in der Stadtregion sogar leicht negativ, ebenso gingen die Wanderungsgewinne 2009 zurück. Betrachtet man die Teilräume getrennt, so ist die Bilanz aus Geburten und Sterbefällen derzeit nur in der Kernstadt Wien positiv und hat dort im Zeitraum 2008 bis 2014 mit gut 16.000 Personen auch deutlich zum Bevölkerungswachstum beigetragen, während die umliegen- den Gemeinden durch eine negative Bilanz mehr als 9.000 Einwohner verloren haben. Deutlich stärker sind die Gewinne jedoch aus der Wanderungsbilanz, wobei wiederum zwei Drittel $(66,1 \%)$ auf die Kernstadt Wien entfallen, das restliche Drittel $(33,9 \%)$ auf das Umland. Durch die hohe Zuwanderung von jungen Menschen und die positive Geburtenbilanz ist Wien mittlerweile auch das jüngste Bundesland Österreichs (Stadt Wien 2014b: 21). 


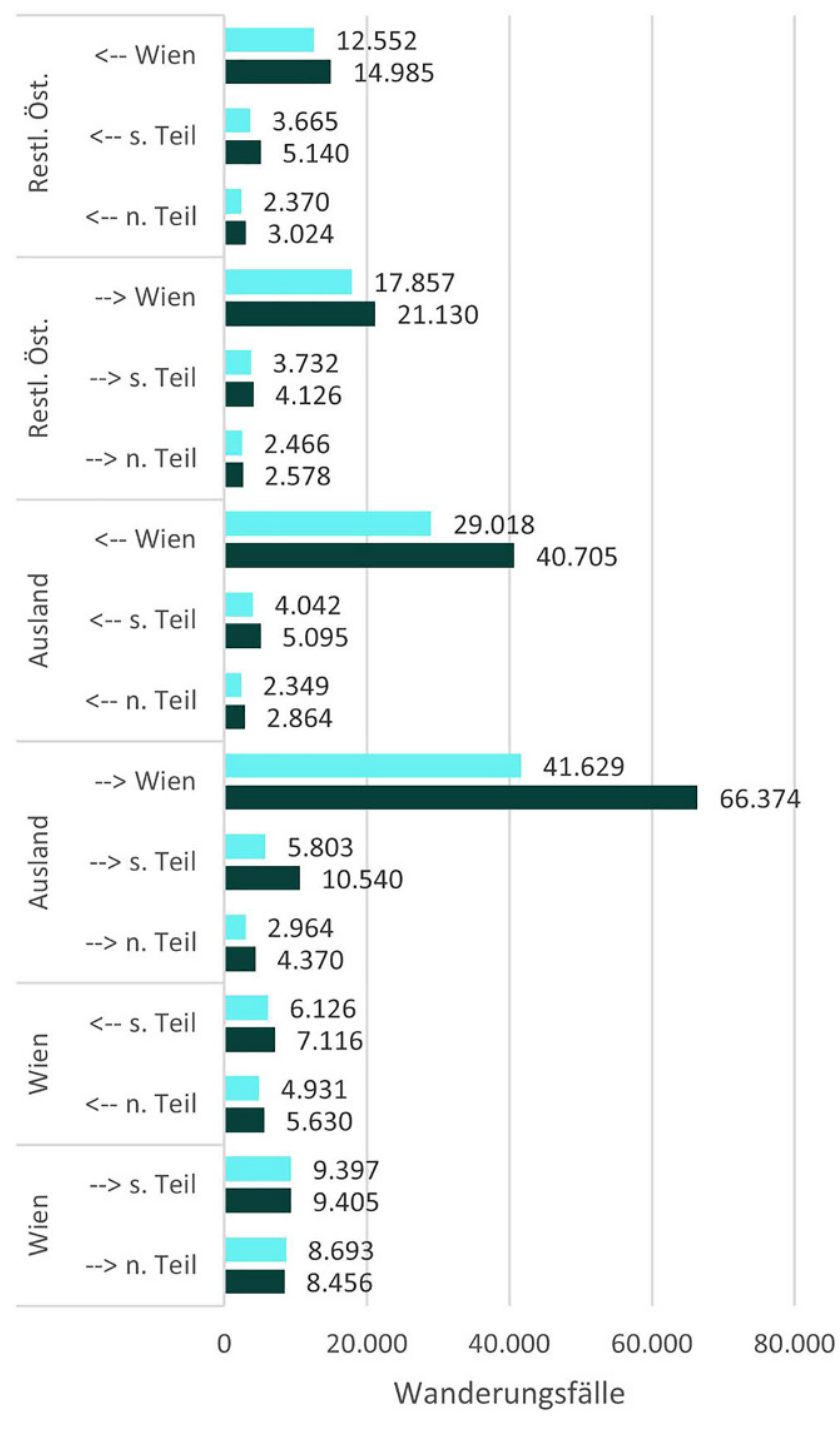

$2007 \square 2014$

Abbildung 6 Wanderungsfälle in der Stadtregion Wien 2007 und 2014. Quelle: Digitales Bevölkerungsregister der Statistik Austria (STATcube), Tabellen „Wanderungen innerhalb Österreichs“ und „Wanderungen mit dem Ausland“; eigene Berechnungen

\subsection{Wanderungsströme}

Die Kernstadt Wien wächst vor allem durch Zuwanderung aus den restlichen Bundesländern Österreichs (beziehungsweise aus Gemeinden im Burgenland und in Niederösterreich außerhalb der Stadtregion) sowie durch internationale Wanderungsbewegungen sowohl aus EU-Ländern als auch aus Drittstaaten. Wanderungsgewinne des Umlands lassen sich größtenteils auf Wanderungsfälle zwischen Kernstadt und Umland, also Suburbanisierung, zurückführen (vgl. Abbildung 6).
Der Vergleich mit dem Jahr $2007^{7}$ zeigt, dass die Wanderungsfälle von Wien in das Umland auf einem ähnlichen Niveau bleiben und Wien hier rund 18.000 Personen pro Jahr im Zuge der Suburbanisierung verliert. Gleichzeitig ziehen jährlich jedoch auch fast 13.000 Personen aus dem Umland zu, bei leicht steigender Tendenz. Die Verluste aus der Suburbanisierung werden folglich durch Wanderungsgewinne aus dem Ausland und aus dem restlichen Österreich ausgeglichen, da Migration aus diesen Regionen hauptsächlich die Kernstadt Wien als Ziel hat.

Die Betrachtung der Altersverteilung der Wanderungsfälle erlaubt einen Rückschluss auf die Form der Migration. Während Ausbildungs- und Erwerbsmigration oftmals in jungem Alter (zwischen 18 und 25 Jahren) zu beobachten ist, kann eine erhöhte Mobilität im Alter zwischen 30 und 40 Jahren in Verbindung mit einer erhöhten Mobilität der unter Vierjährigen auf Familienwanderungen zurückgeführt werden (Bernard/Bell/Charles-Edwards 2014: 215; Gruber 2017: 45). Diese Trends bestätigen sich auch in der Stadtregion Wien. Auch hier ist die Suburbanisierungswanderung nach wie vor zum überwiegenden Teil eine Familienwanderung: Eltern im Alter um die 30 ziehen mit ihren Kindern, die noch nicht im Schulalter sind, in das Umland (vgl. Abbildung 7a). Dies sind die Altersklassen, in denen Wien durchgängig an das Umland Bevölkerung verliert. Im Saldo mit dem restlichen Österreich zeigen sich jedoch deutlich die Wanderungsgewinne im Alter um 20 Jahre, das dem typischen Beginn einer tertiären Ausbildung entspricht. In dieser Altersklasse ergeben sich auch leichte Gewinne mit dem unmittelbaren Umland, was wiederum die Bedeutung Wiens als Ausbildungs- und Studentenstadt unterstreicht (vgl. Musil/Eder 2013). Für einen Vergleich wurden wiederum die Wanderungsdaten von 2007 aus der Bestandsaufnahme (Fassmann/Görgl/Helbich 2009) herangezogen. Hierbei zeigt sich, dass die bis dato beschriebenen Wanderungsbewegungen im Zeitverlauf erstaunlich konstant sind.

Anders gestaltet sich dies jedoch bei der Berücksichtigung internationaler Wanderungen (vgl. Abbildung 7b). Hier werden die Trends aus der Wanderungsbilanz (vgl. Tabelle 2) klar bestätigt, wonach die Wanderungsgewinne aus dem Ausland 2014 auf einem deutlich höheren Niveau liegen als 2007. Wiederum geht der Großteil der internationalen Zuwanderung in die Kernstadt Wien, doch auch die suburbanen Bereiche weisen hier Gewinne aus. Die bestimmenden Trends sind wiederum die Ausbildungs- und Erwerbswanderung von Personen im Alter von 20 bis 30 Jahren. Leichte Verluste gibt es hingegen bei Personen im

\footnotetext{
7 Aufgrund der Datenmenge können nur die Einzeljahre 2007 und 2014 miteinander verglichen werden. Dafür wurden die Daten aus Fassmann/Görgl/Helbich (2009) erneut aufbereitet. Die Daten aus Einzeljahren unterliegen gewissen Schwankungen, dennoch lassen sich durch den Vergleich gewisse Tendenzen und Trends ableiten.
} 


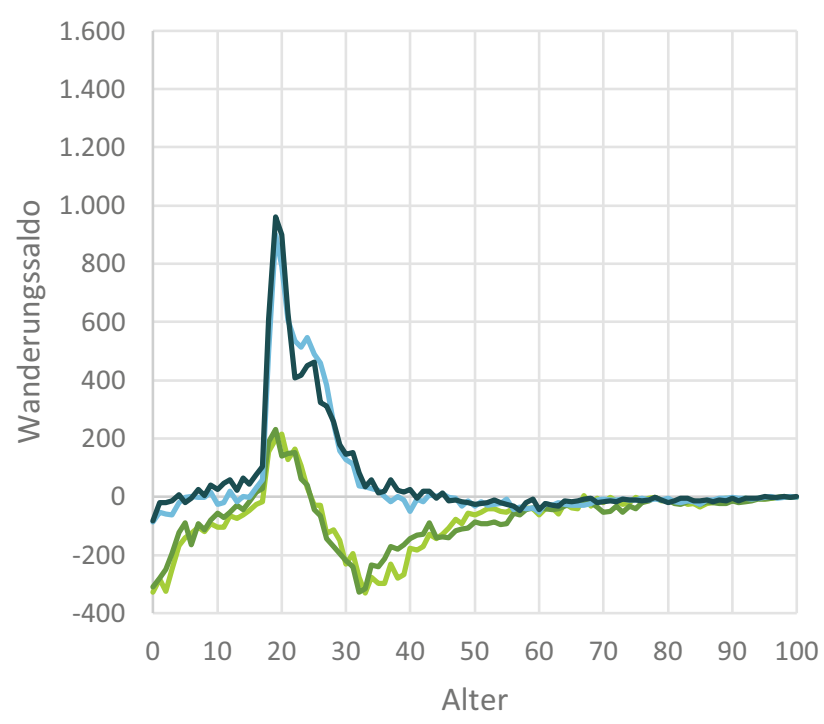

Wien und Umland 2007

—Wien und Umland 2014

_Wien und Restl. Öst. 2007

——Wien und Restl. Öst. 2014

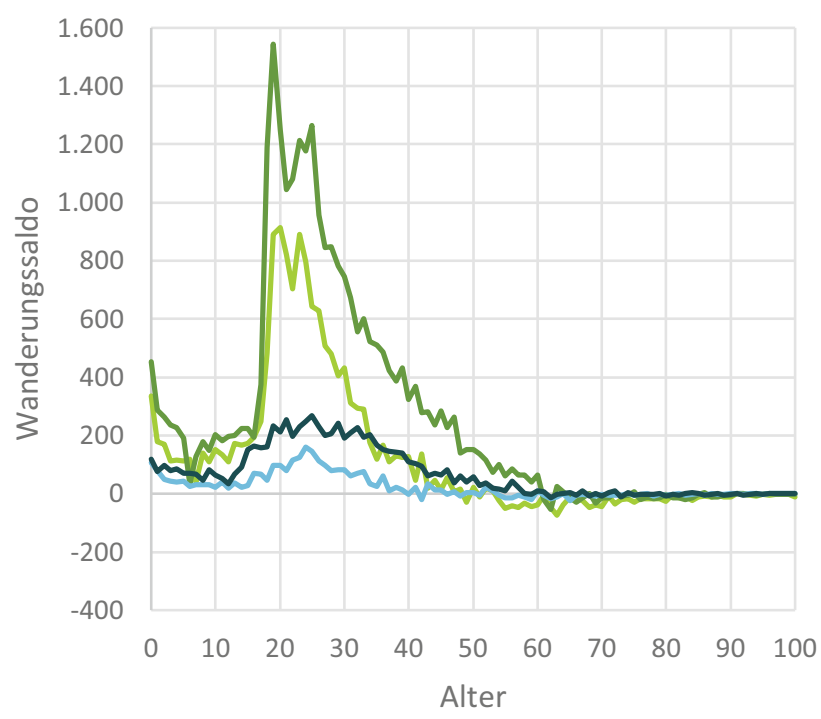

__ Ausland und Wien 2007

_-Ausland und Wien 2014

_ Ausland und Umland 2007

—_Ausland und Umland 2014

Abbildung 7 Wanderungssalden nach Alter hinsichtlich Binnen- und internationaler Wanderung in der Stadtregion Wien. Quelle: Digitales Bevölkerungsregister der Statistik Austria (STATcube), Tabellen „Wanderungen innerhalb Österreichs“ und „Wanderungen mit dem Ausland“; eigene Berechnungen

Ruhestandsalter. Grund dafür könnte sein, dass einige Migranten - beispielsweise ehemalige Gastarbeiter - nach ihrer Pensionierung wieder in ihre Herkunftsländer zurückkehren (Reinprecht 2007).

\section{Siedlungspotenziale und Baulandreserven}

Die bisherigen Analysen des Monitorings haben gezeigt, dass die Stadtregion Wien stark wächst, vor allem aufgrund von Zuwanderung aus dem restlichen Österreich und dem Ausland. In der Kernstadt Wien trägt auch ein Geburtenüberschuss zum Bevölkerungswachstum bei. Folglich stellt sich im Rahmen des Monitorings die aus planungspolitischer Sicht zentrale Frage, nämlich über welche Baulandreserven die Stadtregion verfügt und in welchen Lagen diese noch zu finden sind. Außerdem ist es von Interesse, ob das Wachstum der letzten Jahre in zentralen Orten erfolgt ist und ob hier Handlungsbedarf besteht.

Einen ersten Überblick dazu liefert Abbildung 4d, in der die Siedlungspotenziale (Kernstadt Wien) sowie die Baulandreserven (Umlandgemeinden) dargestellt sind. Größere Reserven sind in der Kernstadt nur noch in den $\mathrm{Au}-$ ßenbezirken (Floridsdorf, Donaustadt, Simmering, Favoriten und Liesing) verfügbar. In Kombination mit den im urbanen Bereich höheren Bebauungsdichten gibt es also auch in Wien somit noch Reserven für größere Bauvorhaben (vgl. Stadt Wien 2014a). In der Innenstadt kann ein relevanter Bevölkerungszuwachs jedoch fast nur über Nachverdichtung (beispielsweise Dachgeschossausbauten, Neubauten mit einer höheren Geschossanzahl, Baulückenschließung) erreicht werden.

Im Umland sind die Baulandreserven höchst unterschiedlich verteilt. Im Wienerwald oder entlang der Donau sind die dafür geeigneten Flächen naturgemäß knapp, in den Gemeinden entlang der Verkehrsachsen, die oft auch von einem hohen Bevölkerungszuwachs gekennzeichnet sind, gibt es aber noch tendenziell größere Reserven. Hier scheinen die Gemeinden ein weiteres Wachstum zu erwarten und dementsprechend verstärkt Flächen als Bauland auszuweisen. Andererseits sind die Reserven auch im Burgenland und damit in vergleichsweise peripheren Gebieten der Stadtregion Wien besonders groß, in denen kein signifikanter Bevölkerungszuwachs zu erwarten ist. Hier gibt es also Anzeichen für einen Baulandüberhang.

\subsection{Analysen mit Hilfe des regionalstatistischen Rasters}

Um die Fragen zu beantworten, ob es einerseits zu einer Konzentration der Bevölkerung oder einer verstärk- 


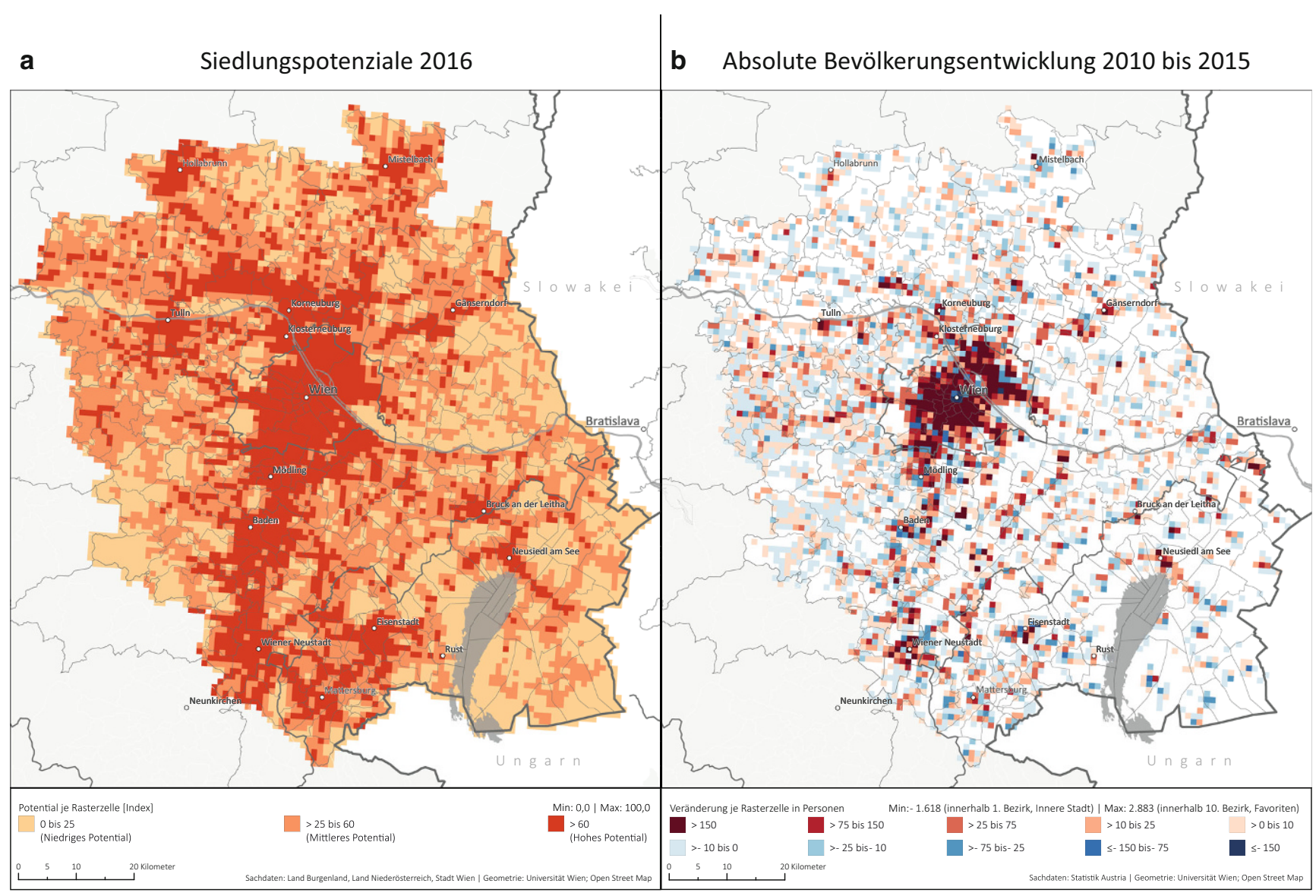

Abbildung 8 Siedlungspotenziale für zukünftige Entwicklungspotenziale der Stadtregion und Bevölkerungsentwicklung in der Stadtregion Wien auf dem geostatistischen Raster (Quelle: Görgl/Eder/Gruber et al. 2017: 84; 106)

ten Siedlungstätigkeit abseits der Hauptorte gekommen ist und ob andererseits die Baulandreserven für die $\mathrm{Zu}$ kunft ausreichend sind, wird, wie in Vorgängerprojekten, auf das regionalstatistische Raster zurückgegriffen (vgl. Fassmann/Görgl/Helbich 2009; Fassmann/Görgl 2010). In Abbildung 8 sind die Siedlungspotenziale 2016, welche zukünftige Entwicklungspotenziale der Bevölkerungsentwicklung darstellen, der tatsächlichen Bevölkerungsentwicklung 2010 bis $2015^{8}$ gegenübergestellt. Dabei zeigt sich, dass aufgrund der hohen Erreichbarkeit und der guten Infrastruktur eine Achse von Süd nach Nord zieht, die von Wiener Neustadt bis nach Mistelbach und Hollabrunn reicht. In den Wienerwaldgemeinden im Westen sowie in den Gemeinden an der slowakischen und ungarischen Grenze sind die Potenziale jedoch geringer. Das Wachstum ist eindeutig auf die Hochpotenzialbereiche konzentriert: Zwischen 2010 und 2015 fanden 94,7 \% des Zuwachses in

\footnotetext{
8 Aufgrund der beschränkten Datenverfügbarkeit kann die Bevölkerungsentwicklung auf dem regionalstatistischen Raster nur zwischen 2010 und 2015 und nicht für den eigentlichen Untersuchungszeitraum 2008 bis 2015 analysiert werden.
}

Hochpotenziallagen und nur 5,3\% außerhalb statt (Görgl/ Eder/Gruber et al. 2017: 112). Vor allem die Kleinstädte weisen im suburbanen Umland das größte Wachstum auf. In den Gebieten zwischen den regionalen Zentren und den Verkehrsachsen geht die Bevölkerung hingegen tendenziell zurück. Das heißt, das Bevölkerungswachstum findet größtenteils in günstigen Lagen statt, die Entwicklung im Umland der Kernstadt Wien deutet auf eine Konzentration der Bevölkerung hin. Dafür ausschlaggebend ist auch die aktuelle Flächenwidmung. So liegen von den derzeit knapp $85,9 \mathrm{~km}^{2}$ gewidmetem Bauland 70,7 \% in Hochpotenzial-, $27,8 \%$ in Mittelpotenzial- und nur $1,5 \%$ in Niedrigpotenziallagen (Görgl/Eder/Gruber et al. 2017: 113).

\subsection{Verfügbare Baulandreserven und Mobilisierungsszenarien}

Gewidmetes Bauland steht in der Regel nicht in vollem Umfang zur Verfügung (vgl. Fassmann/Görgl 2010). Häufig wird davon ausgegangen, dass durch Baulandhortung nur ein Drittel der Fläche für Bauvorhaben unmittelbar verfügbar ist. Um der Frage nachzugehen, ob die Baulandreserven 
Tabelle 3 Potenziell auf Basis der Baulandreserven 2016 mit Wohnraum zu versorgende Personen in der Stadtregion Wien unter Berücksichtigung von Siedlungspotenzialen, Dichteszenarien und Baulandmobilisierung

\begin{tabular}{|c|c|c|c|c|c|c|}
\hline \multirow[b]{2}{*}{$\begin{array}{l}\text { Siedlungs- } \\
\text { potenzial }\end{array}$} & \multirow[b]{2}{*}{$\begin{array}{l}\text { Bauland- } \\
\text { reserven [ha] }\end{array}$} & \multirow[b]{2}{*}{$\begin{array}{l}\text { Bauland- } \\
\text { mobilisierung }\end{array}$} & \multicolumn{4}{|c|}{ Dichteszenarien in Kombination Baulandmobilisierung gestaffelt nach Terzilen } \\
\hline & & & $\begin{array}{l}25 \mathrm{EW} / \mathrm{ha} \\
\text { Freistehende } \\
\text { Einfamilienhäuser }\end{array}$ & $\begin{array}{l}40 \text { EW/ha } \\
\text { Gekuppelte } \\
\text { Einfamilienhäuser }\end{array}$ & $\begin{array}{l}70 \text { EW/ha } \\
\text { Zweigeschossige } \\
\text { Reihenhäuser }\end{array}$ & $\begin{array}{l}150 \text { EW/ha } \\
\text { Geschosswohnbau }\end{array}$ \\
\hline Hoch & $6.777,74$ & \multirow{4}{*}{$\begin{array}{l}1 . \\
(33,3 \%)\end{array}$} & 56.481 & 90.370 & 158.147 & 338.887 \\
\hline Mittel & $1.680,67$ & & 14.006 & 22.409 & 39.216 & 84.034 \\
\hline Niedrig & 130,12 & & 1.084 & 1.735 & 3.036 & 6.506 \\
\hline Gesamt & $\mathbf{8 . 5 8 8 , 5 3}$ & & 71.571 & 114.514 & 200.399 & 429.426 \\
\hline Hoch & $6.777,74$ & \multirow{4}{*}{$\begin{array}{l}2 . \\
(66,6 \%)\end{array}$} & 112.962 & 180.740 & 316.294 & 677.774 \\
\hline Mittel & $1.680,67$ & & 28.011 & 44.818 & 78.431 & 168.067 \\
\hline Niedrig & 130,12 & & 2.169 & 3.470 & 6.072 & 13.012 \\
\hline Gesamt & $8.588,53$ & & 143.142 & 229.027 & 400.798 & 858.853 \\
\hline Hoch & $6.777,74$ & \multirow{4}{*}{$\begin{array}{l}3 . \\
(100,0 \%)\end{array}$} & 169.443 & 271.109 & 474.442 & 1.016 .660 \\
\hline Mittel & $1.680,67$ & & 42.017 & 67.227 & 117.647 & 252.101 \\
\hline Niedrig & 130,12 & & 3.253 & 5.205 & 9.108 & 19.518 \\
\hline Gesamt & $8.588,53$ & & 214.713 & 343.541 & 601.197 & 1.288.279 \\
\hline
\end{tabular}

unter verschiedenen Mobilisierungsannahmen ausreichend sind, wird in Tabelle 3 (wie in diversen Vorgängerstudien) das gewidmete Bauland in der Stadtregion Wien mit diversen Dichteszenarien verschnitten, die vom Salzburger Institut für Raumordnung und Wohnen (2007: 9) für den österreichischen Kontext definiert wurden. In einem konservativen Szenario, das von einer Baulandmobilisierung von einem Drittel ausgeht, zeigt sich durchaus eine Baulandknappheit. Unter diesen Annahmen könnten nur gut 56.000 zusätzliche Einwohner in Einfamilienhäusern auf Hochpotenzialflächen untergebracht werden. Vor dem Hintergrund des raschen Bevölkerungswachstums in der Stadtregion wird also klar, dass der Fokus auf dichtere Bebauungsformen in der Stadtregion - auch in den Umlandgemeinden - unumgänglich ist. Dazu kommt die Notwendigkeit einer weiteren Nachverdichtung in den Ortskernen.

Weiters unterstreicht die Analyse, dass es nach wie vor große unbebaute Baulandreserven in Hochpotenziallagen gibt, nämlich $86,9 \mathrm{~km}^{2}$. Stünde das gesamte Bauland unmittelbar zur Verfügung und würde es mit Geschosswohnbau (150 Einwohner/ha) bebaut, wäre Wohnraum für fast 1,3 Millionen zusätzliche Personen vorhanden. Selbstverständlich eignet sich nicht jede Parzelle für diese Bebauungsdichte, teilweise würde dies auch der vorliegenden Flächenwidmung widersprechen. Durch die hypothetische Berechnung kann aber gezeigt werden, dass das Potenzial des derzeit gewidmeten Baulands groß ist. Zusätzlichem Bevölkerungswachstum sollte folglich nicht nur mit neuer Baulandausweisung begegnet werden, sondern auch mit konsequenten Maßnahmen zur Baulandmobilisierung.

\section{Zusammenfassung und Diskussion}

Die entwickelte Methodik des PGO-Projekts „Monitoring der Siedlungsentwicklung in der Stadtregion: Strategien zur räumlichen Entwicklung der Ostregion" hat sich insofern in der Praxis bewährt, als dass sich mit relativ wenigen Parametern (Bevölkerungsentwicklung, Bilanz der Geburten und Sterbefälle, Wanderungsbilanz, Wanderungsströme und Baulandreserven) ein detailliertes Bild zur Bevölkerungsund Siedlungsentwicklung einer Stadtregion zeichnen lässt. Dadurch lassen sich bei überschaubarem Ressourceneinsatz die zentralen Fragestellungen für die räumliche Entwicklung einer Stadtregion beantworten, woraus sich konkreter Handlungsbedarf und Handlungsempfehlungen ergeben. Im vorliegenden Beitrag wurden die Methodik und in Grundzügen die Ergebnisse des quantitativen Teils des Monitorings vorgestellt, aber auch eine zusätzliche Auswertung hinsichtlich Baulandmobilisierung und Bebauungsdichte durchgeführt.

Der Fokus des vorliegenden Beitrags wurde auf die quantitative Untersuchung gelegt, um zu zeigen, dass die Datenverfügbarkeit in Österreich durch die Einführung des zentralen Melderegisters und des darauf basierenden Bevölkerungsregisters und der Wanderungsstatistik mittlerweile eine sehr hohe Qualität erreicht hat. Somit lassen sich auch mit quantitativen Daten kleinräumig aussagekräftige Ergebnisse erzielen, wodurch eine genauere Analyse der Stadtregion Wien möglich wird. Das regionalstatistische Raster ermöglicht als zusätzliche Analyseeinheit eine kleinräumige und somit treffsichere Analyse der Siedlungsentwicklung. Damit können Aussagen über die Siedlungsentwicklung und die Zunahme des Flächenverbrauchs getätigt werden. Durch das zentrale Melderegister stehen grundlegende Bevölkerungsdaten auch regelmäßig und nach einer kurzen 
Frist zur Verfügung. Somit ist die notwendige Datenbasis für ein Monitoring unmittelbar verfügbar. Ein Monitoring stellt aber immer nur eine Momentaufnahme dar, wodurch eine regelmäßige Durchführung wichtig und sinnvoll erscheint, um eine entsprechende Planungsgrundlage auf dem neuesten Stand zu erhalten. In diesem Zusammenhang ist ein übersichtliches Variablenset mit schnell zu berechnenden Indikatoren von Vorteil. Tiefergehende, spezialisierte Analysen werden dadurch aber nicht abgelöst, sondern sollten punktuell zusätzlich vorgenommen werden.

Das Monitoring der Stadtregion Wien im Zeitraum 2008 bis 2015 bestätigt, dass die Stadtregion deutlich schneller gewachsen ist als erwartet. Die Dynamik des Wachstums hat seit der letzten Untersuchungsperiode damit deutlich zugenommen. Die Kernstadt Wien wächst zwar auch aufgrund der positiven Geburtenbilanz, insgesamt betrachtet sind jedoch die Bevölkerungsgewinne aus der Binnenwanderung und vor allem aus der internationalen Migration der entscheidende Wachstumsfaktor in der Stadtregion. Das Muster der Wanderungsströme hat sich seit dem Jahr 2008 deutlich diversifiziert. Neben den alten EU-Mitgliedstaaten steigen die Migrationsbewegungen auch aus den osteuropäischen Staaten, die 2004, 2007 und 2013 der Europäischen Union (EU) beigetreten sind. Daneben ist auch die Zuwanderung aus Drittstaaten auf einem hohen Niveau. Die internationale Zuwanderung trägt mittlerweile am stärksten zum Wachstum der Stadtregion bei.

Während die internationale Wanderung zunimmt, bleibt die Suburbanisierung auf einem ähnlichen Niveau wie in früheren Jahren. Dennoch gibt es auch hier neue Trends und Tendenzen zu beobachten. Neben der Kernstadt Wien konzentriert sich Zuwanderung auch im suburbanen Raum mittlerweile vermehrt in zentraleren Gemeinden (Kleinstädte, regionale Zentren). Die verstärkte Bautätigkeit abseits der Achsen, die im Jahr 2008 beobachtet werden konnte, ist zurückgegangen. Die bekannten Muster der Ausbildungs-, Erwerbs- und Familienwanderung (vgl. Bernard/Bell/CharlesEdwards 2014) können in der Stadtregion Wien jedoch weiterhin beobachtet werden.

Diese Entwicklungen haben gezeigt, dass auch in Wien eine stärkere Betrachtung der Reurbanisierung sinnvoll ist. Die Stadt wächst innerhalb der administrativen Grenzen, und zwar sowohl in den Stadtentwicklungsgebieten in den Flächenbezirken als auch in den inneren Bereichen. Die Stadt Wien versucht, diese Entwicklung auch aktiv im Rahmen der Stadtentwicklungspolitik zu gestalten (Stadt Wien 2014b). Suburbanisierung lässt sich aber nach wie vor beobachten und stellt folglich nicht wie im Modell von van den Berg, Drewett, Klaassen et al. (1982: 36) eine vorübergehende Phase der Stadtentwicklung dar. In der Stadtregion Wien lassen sich in den letzten Jahren dabei auch neue Formen der Suburbanisierung beobachten, wie beispielsweise die transnationale Suburbanisierung mit Bratislava, die durch die Überlappung der beiden Stadtregionen begründet ist (vgl. Hardi 2012; Sohn/Giffinger 2015).

Die Ziele der Standortentwicklung in der Stadtregion haben sich seit der letzten Untersuchung auch kaum geändert. Durch das starke Bevölkerungswachstum der letzten Jahre sind die Herausforderungen nicht weniger geworden, sondern haben sich teilweise in ihrer Dynamik verstärkt. Das Monitoring hat gezeigt, dass die Ziele, die im Leitbild der strukturierten Stadtregion Wien formuliert wurden (Planungsgemeinschaft Ost 2011), größtenteils erfüllt wurden beziehungsweise werden. Dazu zählen beispielsweise die weitere Verdichtung in regionalen Entwicklungszentren und der nachhaltigere Umgang mit Baulandreserven. Dafür sind jedoch nicht unbedingt die vorhandenen Planungsinstrumente ausschlaggebend, sondern auch Marktmechanismen (etwa steigende Bodenpreise) oder steigender Leidensdruck in den Gemeinden, der zu einer interkommunalen Kooperation zwingt. Die zunehmende Baulandknappheit zeichnet sich nun deutlich ab, ein sparsamer Umgang mit den Reserven ist unumgänglich. Durch eine zunehmende Nachfrage und Dichte steigen auch die Verkehrsbelastung und der Bedarf an leistbarem Wohnraum. Vor allem der letzte Punkt wird in Zukunft noch wichtiger werden, da Flächen für große innerstädtische Wohnbauprojekte wie am neuen Hauptbahnhof, am Nordbahnhof oder in der Seestadt Aspern, die in den letzten Jahren bebaut wurden, rar werden (vgl. Stadt Wien 2014a).

Im Rahmen des vorliegenden Beitrags wurde zur genaueren Betrachtung der Siedlungsentwicklung der Flächenverbrauch in diversen Szenarien zur Baulandmobilisierung verdeutlicht. Dadurch zeigt sich, dass die gewidmeten Baulandreserven in der Stadtregion bei der üblicherweise niedrigen Baulandmobilisierung für die zukünftige Siedlungsentwicklung nur dann ausreichend sind, wenn mit einer höheren Dichte gebaut wird. Bei einer ausschließlichen Bebauung mit Einfamilienhäusern kann durchaus eine zukünftige Baulandknappheit in den Hochpotenzialregionen prognostiziert werden. Die derzeitigen Entwicklungen haben aber gezeigt, dass durch steigende Nachfrage und Bodenpreise der Trend zur Verdichtung auch ohne raumordnungspolitische Maßnahmen zugenommen hat. Die größte Problematik in diesem Bereich stellt weiterhin die geringe Verfügbarkeit des gewidmeten Baulandes aufgrund von Baulandhortung dar. Dadurch leistet der Beitrag einen ersten Schritt zur Operationalisierung von Siedlungsentwicklung und potenziellem Flächenverbrauch.

Abschließend lässt sich aus den Analysen schließen, dass die Stadtregion Wien aus planerischer Perspektive als eine miteinander verbundene Einheit zu betrachten ist, denn funktionale und strukturelle Zusammenhänge werden stetig größer. Auch wenn nicht alle Städte und Gemeinden in der Stadtregion Wien gleichermaßen stark wachsen, stellt der kontinuierliche Bevölkerungszuwachs heute eine gemein- 
same Herausforderung dar, welche die Bereitschaft zur Kooperation erhöhen könnte. Gerade in den letzten Jahren hat es in der Stadtregion vor allem in Hinblick auf Umsetzungsmaßnahmen und konkrete länder- und gemeindeübergreifende Projekte einige Fortschritte gegeben (vgl. Görgl/ Eder/Gruber et al. 2017). Wie in den allermeisten Fällen ist das bis zu einem bestimmten Grad dem steigenden Leidensund Planungsdruck geschuldet. Idealerweise sollte sich dies folglich auch in einem gemeinsamen und bestenfalls auch verbindlichen Plandokument niederschlagen.

\section{Handlungsempfehlungen}

Der vorliegende Beitrag hatte zum Ziel, die Methodik und die grundlegenden Ergebnisse des PGO-Projekts „Monitoring der Siedlungsentwicklung in der Stadtregion: Strategien zur räumlichen Entwicklung der Ostregion" der Stadtregion Wien vorzustellen und diese zu diskutieren. Daraus können in einem abschließenden Schritt Handlungsoptionen abgeleitet werden, die bei der Erstellung von zukünftigen Stadtregion-Monitorings beachtet werden können.

Erstens stellt die Verwendung neuer, registerbasierter Daten einen Mehrwert für die Treffsicherheit des Monitorings dar. Auch wenn der ausschließliche Einsatz von quantitativen Datensätzen grundsätzlich hinterfragt werden kann, belegt der vorliegende Beitrag, dass Sekundärdaten und deren Auswertungsmöglichkeiten sich zunehmend weiterentwickeln und diese auch eingesetzt werden sollten. Eine Verknüpfung von quantitativen und qualitativen Forschungsansätzen hat sich im Rahmen des Forschungsprojekts zudem ebenfalls bewährt.

Zweitens belegt der Beitrag, dass zur Veranschaulichung der Ergebnisse die Entwicklung von Szenarien hilfreich ist. Der Status quo alleine ist zwar eine gute Planungsgrundlage, die Berücksichtigung leicht nachvollziehbarer Waswäre-wenn-Szenarien stellt jedoch eine gute Ergänzung dar, mit Hilfe derer besser abgeschätzt werden kann, ob Handlungsbedarf besteht. Die Szenarien können einen solchen gegebenenfalls auch leicht vermitteln. Die Verknüpfung verschiedener kleinräumiger Datenquellen ist für den Entwurf von Szenarien besonders lohnend.

Drittens wird klar, dass die isolierte Beobachtung einzelner Stadtregionen in Forschungsprojekten oftmals dazu führt, dass ein Verständnis für grundlegende, internationale Entwicklungen bei den Entscheidungsträgern fehlt. Zwar ist die Analyse der Stadt Wien über ihre administrativen Grenzen hinaus schon ein erster Schritt in Richtung einer integrierten Betrachtung. Allerdings sollte auch bei regionalen Projekten ein größerer Wert darauf gelegt werden, die Ergebnisse in den internationalen Forschungsdiskurs einzubetten. So lassen sich regionale Trends besser verstehen, und internationale Vergleiche können helfen, Folgen und
Effekte von Entwicklungen besser einzuschätzen. Auch die intensivere Beschäftigung mit international erfolgreichen Governance-Ansätzen kann für die involvierten Fachplanungsebenen und politischen Akteure ein wertvolles Mittel sein, um für die eigene Stadtregion nachhaltige Lösungsansätze zu entwickeln.

Förderung Die Finanzierung und Beauftragung des Projekts erfolgte durch die Planungsgemeinschaft Ost (PGO).

\section{Literatur}

Banerjee, T. (1996): Role of Indicators in Monitoring Growing Urban Regions. The Case of Planning in India's National Capital Region. In: Journal of the American Planning Association 62, 2, 222-235. doi: 10.1080/01944369608975686

Bernard, A.; Bell, M.; Charles-Edwards, E. (2014): Life-Course Transitions and the Age Profiles of Internal Migration. In: Population and Development Review 40, 2, 213-239.

Brake, K.; Herfert, G. (Hrsg.) (2012): Reurbanisierung. Materialität und Diskurs in Deutschland. Wiesbaden.

Buzar, S.; Hall, R.; Ogden, P. E. (2007): Beyond gentrification: the demographic reurbanisation of Bologna. In: Environment and Planning A 39, 1, 64-85. doi: 10.1068/a39109

Champion, T. (2001): Urbanization, Suburbanization, Counterurbanization and Reurbanization. In: Paddison, R. (Hrsg.): Handbook of Urban Studies. London, 143-161.

Cheshire, P. (1995): A new phase of urban development in Western Europe? The evidence for the 1980s. In: Urban Studies 32, 7, 10451063. doi: 10.1080/00420989550012564

Döringer, S.; Görgl, P.; Huemer, J. (2014): Standort- und Verdichtungspotenziale im Nahbereich von Bahnhöfen und Haltestellen in der Stadtregion plus. Wien.

Fassmann, H.; Görgl, P. (2010): Wachsende Stadtregion - Modellrechnungen zum Bevölkerungswachstum in der Stadtregion Ost. In: Mitteilungen der Österreichischen Geographischen Gesellschaft 152, 183-200.

Fassmann, H.; Görgl, P.; Helbich, M. (2009): Atlas der wachsenden Stadtregion. Materialband zum Modul I des Projekts „Strategien zur räumlichen Entwicklung der Ostregion (SRO)“ im Auftrag der Planungsgemeinschaft Ost (PGO). Wien.

Fassmann, H.; Hatz, G. (2009): Wien. Städtebauliche Entwicklung und planerische Probleme. In: Fassmann, H.; Hatz, G.; Matznetter, W. (Hrsg.): Wien - Städtebauliche Strukturen und gesellschaftliche Entwicklungen. Wien, 13-35.

Fassmann, H.; Münz, R. (1995): Einwanderungsland Österreich? Historische Migrationsmuster, aktuelle Trends und politische Maßnahmen. Wien.

Giffinger, R.; Hamedinger, A. (2009): Metropolitan competitiveness reconsidered. The role of territorial capital and metropolitan governance. In: Terra Spectra - Planning Studies - Central European Journal of Spatial and Landscape Planning 20, 1, 3-12.

Giffinger, R.; Kalasek, R.; Wonka, E. (2006): Ein neuer Ansatz zur Abgrenzung von Stadtregionen: methodische Grundlagen und Perspektiven zur Anwendung. In: Schrenk, M. (Hrsg.): Sustainable Solutions for the Information Society. Proceedings of 11th International Conference on Urban Planning and Spatial Development in the Information Society. Wien, 677-683.

Görgl, P. (2008): Die Amerikanisierung der Wiener Suburbia? Der Wohnpark Fontana. Eine sozialgeographische Studie. Wiesbaden. doi: 10.1007/978-3-531-90965-3

Görgl, P.; Eder, J.; Gruber, E.; Fassmann, H. (2017): Monitoring der Siedlungsentwicklung in der Stadtregion ${ }^{+}$. Strategien zur räumlichen Entwicklung der Ostregion. Wien. 
Görgl, P.; Gruber, E. (2015): Die kooperative Stadt der Zukunft? StadtUmland-Zusammenarbeit am Beispiel der Stadtregion Wien. In: Fritz, J.; Tomaschek, N. (Hrsg.): Die Stadt der Zukunft. Aktuelle Trends und zukünftige Herausforderungen. Münster, 267-280. = University Society Industry 4.

Görgl, P.; Helbich, M.; Matznetter, W.; Fassmann, H. (2011): Spatial and Social Development Trends of Metropolitan Vienna: An Overview. In: Szirmai, V. (Hrsg.): Urban Sprawl in Europe. Similarities or differences? Budapest, 107-140.

Gruber, E. (2017): Im Ruhestand aufs Land? Ruhestandsmigration und deren Bedeutung für ländliche Räume in Österreich. Berlin.

Hanika, A. (2006): Aktualisierung der regionalisierten ÖROK-Bevölkerungs-, Erwerbstätigen- und Haushaltsprognose 2001 bis 2031. Wien.

Hanika, A.; Fuchs, R.; Klotz, J. (2015): ÖROK-Regionalprognosen 2014-2030. Teil 1: Bevölkerung. Wien.

Hardi, T. (2012): Cross-Border Suburbanisation: The Case of Bratislava. In: Csapó, T.; Balogh, A. (Hrsg.): Development of the Settlement Network in the Central European Countries: Past, Present, and Future. Berlin/Heidelberg, 193-206.

Helbich, M. (2012): Beyond Postsuburbia? Multifunctional Service Agglomeration in Vienna's Urban Fringe. In: Tijdschrift voor Economische en Sociale Geografie 103, 1, 39-52. doi: 10.1111/j.1467-9663.2011.00673.x

Helbich, M.; Leitner, M. (2009): Spatial Analysis of the Urban-to-Rural Migration Determinants in the Viennese Metropolitan Area. A Transition from Suburbia to Postsuburbia? In: Applied Spatial Analysis and Policy 2, 3, 237-260. doi: 10.1007/s12061-0099026-8

Helbich, M.; Leitner, M. (2010): Postsuburban Spatial Evolution of Vienna's Urban Fringe: Evidence from Point Process Modeling. In: Urban Geography 31, 8, 1100-1117. doi: 10.2747/02723638.31.8.1100

Helbrecht, I. (2001): Postmetropolis: Die Stadt als Sphinx. In: Geographica Helvetica 56, 3, 214-222.

Herfert, G.; Osterhage, F. (2012): Wohnen in der Stadt: Gibt es eine Trendwende zur Reurbanisierung? Ein quantitativ-analytischer Ansatz. In: Brake, K.; Herfert, G. (Hrsg): Reurbanisierung. Materialität und Diskurs in Deutschland. Wiesbaden, 86-112.

Hirschle, M.; Schürt, A. (2010): Wanderungstrends in Stadtregionen - Neben Suburbanisierung auch Reurbanisierung? In: DittrichWesbuer, A.; Knapp, W.; Osterhage, F. (Hrsg): Postsuburbanisierung und die „Renaissance der (Innen-)Städte“. Detmold. 27-43. $=$ Metropolis und Region 6 .

Kontgis, C.; Schneider, A.; Fox, J.; Saksena, S.; Spencer, J. H.; Castrence, M. (2014): Monitoring peri-urbanization in the greater Ho Chi Minh City metropolitan area. In: Applied Geography 53, 9, 377-388. doi: 10.1016/j.apgeog.2014.06.029

Lever, W. F. (1993): Reurbanisation - The Policy Implications. In: Urban Studies 30, 2, 267-284. doi: 10.1080/00420989320080281

Musil, R.; Eder, J. (2013): Wien und seine Hochschulen. Regionale Wertschöpfungseffekte der Wiener Hochschulen. Wien. = ISRForschungsbericht 40. doi: 10.1553/ISR_FB040
Ogden, P. E.; Hall, R. (2000): Households, Reurbanisation and the Rise of Living Alone in the Principal French Cities, 1975-90. In: Urban Studies 37, 2, 367-390. doi: 10.1080/0042098002230

Oswalt, P.; Rieniets, T. (2006): Atlas of Shrinking Cities. Ostfildern.

Othengrafen, F.; Schmidt-Lauber, B.; Hannemann, C.; Pohlan, J.; Roost, F. (Hrsg.) (2016): Jahrbuch StadtRegion 2015/2016. Schwerpunkt: Planbarkeiten. Leverkusen.

Planungsgemeinschaft Ost (2011): stadtregion+. Planungskooperation zur räumlichen Entwicklung der Stadtregion Wien, Niederösterreich, Burgenland. Zwischenbericht. Wien.

Pohlan, J.; Strote, J. (2017): Cities under observation: Social monitoring in integrated neighbourhood development in Hamburg. In: Procedia Computer Science 112, 2426-2434. doi: 10.1016/j.procs.2017.08.175

Reinprecht, C. (2007): Alt nach der Gastarbeit. In: Fassmann, H. (Hrsg.): 2. Österreichischer Migrations- und Integrationsbericht 2001-2006. Rechtliche Rahmenbedingungen, demographische Entwicklungen, sozioökonomische Strukturen. Klagenfurt, 211224.

Rérat, P. (2012): The New Demographic Growth of Cities: The Case of Reurbanisation in Switzerland. In: Urban Studies 49, 5, 11071125. doi: 10.1177/0042098011408935

Salzburger Institut für Raumordnung und Wohnen (2007): Infrastrukturkostenstudie Salzburg. Zusammenhänge von Bebauungsart und -dichte sowie Erschließungskosten. Salzburg. = SIR Konkret 4.

Sassen, S. (1994): Cities in a World Economy. Thousand Oaks.

Sohn, C.; Giffinger, R. (2015): A Policy Network Approach to CrossBorder Metropolitan Governance: The Cases of Vienna and Bratislava. In: European Planning Studies 23, 6, 1187-1208. doi: 10.1080/09654313.2014.994089

Soja, E. (2000): Postmetropolis: Critical Studies of Cities and Regions. Oxford.

Stadt Wien (2014a): STEP 2025 - Stadtentwicklungsplan - Mut zur Stadt. Wien.

Stadt Wien (2014b): Wien wächst ... Bevölkerungsentwicklung in Wien und den 23 Gemeinde- und 250 Zählbezirken. Wien.

Suitner, J.; Krisch, A.; Pühringer, F. (2018): TRANS[form]DANUBIEN. Eine urbane Metamorphologie der Wiener Stadtplanung anhand der Entwicklungsdynamik Wiens links der Donau. Abschlussbericht zum Forschungsprojekt. Wien.

van den Berg, L.; Drewett, R.; Klaassen, L.; Rossi, A.; Vijverberg, C. (1982): Urban Europe. A Study of Growth and Decline. Oxford.

Weigl, A. (2003): „Unbegrenzte Großstadt“ oder „Stadt ohne Nachwuchs"? Zur demographischen Entwicklung Wiens im 20. Jahrhundert. In: Eder, F. X.; Eigner, P.; Resch, A.; Weigl, A. (Hrsg.): Wien im 20. Jahrhundert. Wirtschaft, Bevölkerung, Konsum. Innsbruck, 141-200. = Querschnitte 12 .

Weiland, U.; Richter, M. (2008): Monitoring und Evalulation der Stadtentwicklung. In: CONTUREC 3, 5-14.

Yueliang, M.; Ruisong, X. (2010): Remote sensing monitoring and driving force analysis of urban expansion in Guangzhou City, China. In: Habitat International 34, 2, 228-235. https://doi.org/10.1016/j. habitatint.2009.09.007 